\title{
Genetic variation affects morphological retinal phenotypes extracted from UK Biobank optical coherence tomography images
}

\author{
Hannah Currant $\oplus^{1 *}$, Pirro Hysi $\oplus^{2,3}$, Tomas W. Fitzgerald $\oplus^{1}$, Puya Gharahkhani $\oplus^{4}$, Pieter \\ W. M. Bonnemaijer ${ }^{5,6,7}$, Anne Senabouth $\oplus^{8}$, Alex W. Hewitt ${ }^{9,10}$, UK Biobank Eye and \\ Vision Consortium, International Glaucoma Genetics Consortium, Denize Atan ${ }^{11,12}$, \\ Tin Aung ${ }^{13,14,15}$, Jason Charng ${ }^{16}{ }^{16}$, Hélène Choquet ${ }^{17}{ }^{17}$, Jamie Craig ${ }^{18}$, Peng T. Khaw ${ }^{19}$, \\ Caroline C. W. Klaver $\oplus^{5,6,20,21}$, Michiaki Kubo ${ }^{22}$, Jue-Sheng Ong ${ }^{4}$, Louis \\ R. Pasquale ${ }^{23}$, Charles A. Reisman ${ }^{24}$, Maciej Daniszewski $\mathbb{D}^{25}$, Joseph E. Powell ${ }^{8,26}$, \\ Alice Pébay ${ }^{25,27}$, Mark J. Simcoe $\varpi^{28,29}$, Alberta A. H. J. Thiadens $\varpi^{5}$, Cornelia M. van \\ Duijn $\oplus^{30}$, Seyhan Yazar $\oplus^{31}$, Eric Jorgenson ${ }^{17}{ }^{17}$, Stuart MacGregor $\oplus^{4}$, Chris \\ J. Hammond $\oplus^{2}$, David A. Mackey $\oplus^{16}$, Janey L. Wiggs $\oplus^{32}$, Paul J. Foster $\oplus^{19}$, Praveen \\ J. Patel ${ }^{19}{ }^{19}$, Ewan Birney ${ }^{1 \neq}$, Anthony P. Khawaja ${ }_{\mathbb{D}}^{19 \neq *}$
}

1 European Molecular Biology Laboratory, European Bioinformatics Institute, Wellcome Genome Campus, Cambridge, United Kingdom, 2 School of Life Course Sciences, Section of Ophthalmology, King's College London, London, United Kingdom, 3 Department of Twin Research and Genetic Epidemiology, King's College London, London, United Kingdom, 4 Statistical Genetics, QIMR Berghofer Medical Research Institute, Brisbane, Australia, 5 Department of Ophthalmology, Erasmus MC, Rotterdam, The Netherlands, 6 Department of Epidemiology, Erasmus MC, Rotterdam, The Netherlands, 7 The Rotterdam Eye Hospital, Rotterdam, The Netherlands, 8 Garvan Weizmann Centre for Cellular Genomics, Garvan Institute of Medical Research, The Kinghorn Cancer Centre, Darlinghurst, Australia, 9 Menzies Institute for Medical Research, School of Medicine, University of Tasmania, Tasmania, Australia, 10 Centre for Eye Research Australia, Royal Victorian Eye and Ear Hospital, East Melbourne, Australia, 11 Translational Health Sciences, Bristol Medical School, University of Bristol, Bristol, United Kingdom, 12 Bristol Eye Hospital, University Hospitals Bristol \& Weston NHS Foundation Trust, Bristol, United Kingdom, 13 Singapore Eye Research Institute, Singapore National Eye Centre, Singapore, 14 Ophthalmology \& Visual Sciences Academic Clinical Program, Duke-NUS Medical School, Singapore, 15 Department of Ophthalmology, Yong Loo Lin School of Medicine, National University of Singapore, Singapore, 16 Centre for Ophthalmology and Visual Science, Lions Eye Institute, The University of Western Australia, Perth, Australia, 17 Kaiser Permanente Northern California Division of Research, Oakland, California, United States of America, 18 Department of Ophthalmology, Flinders University, Flinders Medical Centre, Bedford Park, Australia, 19 NIHR Biomedical Research Centre, Moorfields Eye Hospital NHS Foundation Trust and UCL Institute of Ophthalmology, London, United Kingdom, 20 Department of Ophthalmology Radboud University Medical Center, Nijmegen, The Netherlands, 21 Institute of Molecular and Clinical Ophthalmology, Basel, Switzerland, 22 RIKEN Center for Integrative Medical Sciences, Yokohama, Japan, 23 Eye and Vision Research Institute, Icahn School of Medicine at Mount Sinai, New York, New York, United States of America, 24 Topcon Healthcare Solutions R\&D, Oakland, New Jersey, United States of America, 25 Department of Anatomy and Physiology, The University of Melbourne, Parkville, Australia, 26 UNSW Cellular Genomics Futures Institute, University of New South Wales, Sydney, Australia, 27 Department of Surgery, The University of Melbourne, Parkville, Australia, 28 Department of Ophthalmology, Kings College London, London, United Kingdom, 29 Institute of Ophthalmology, University College London, London, United Kingdom, 30 Nuffield Department Of Population Health, University of Oxford, Big Data Institute, Li Ka Shing Centre for Health Information and Discovery, Oxford, United Kingdom, 31 Garvan-Weizmann Centre for Single Cell Genomics, Garvan Institute of Medical Research, Sydney, Australia, 32 Department of Ophthalmology, Harvard Medical School, Massachusetts Eye and Ear, Boston, Massachusetts, United States of America

‡ EB and APK jointly supervised this work.

* currant@ebi.ac.uk (HC); anthony.khawaja@ucl.ac.uk (APK)

Data Availability Statement: The genetic and phenotypic UK Biobank data are available upon application to the UK Biobank (https://www. ukbiobank.ac.uk). The summary statistics are available to download from the GWAS catalog (https://www.ebi.ac.uk/gwas/home, GCST90014266 and GCST90014267 for RNFL and GCIPL, respectively).

Funding: $\mathrm{H}$ Currant and $\mathrm{EB}$ are funded by EMBL. APK was supported by a UK Research \& 
Innovation Future Leaders Fellowship, a Moorfields Eye Charity Career Development Fellowship, and an Alcon Research Institute Young Investigator Award. The Rotterdam Study is funded by Erasmus MC and Erasmus University, Rotterdam, Netherlands Organization for the Health Research and Development (ZonMw), the Research Institute for Diseases in the Elderly (RIDE), the Ministry of Education, Culture and Science, the Ministry for Health, Welfare and Sports, the European Commission (DG XII), and the Municipality of Rotterdam. PG is supported by a NHMRC Investigator Grant (\#1173390). SM acknowledges Australian National Health and Medical Research Council grants 1154543, 1150144 and 1116360. LRP is funded by NIH EY015473 and also supported by a Challenge Grant from Research to Prevent Blindness, NYC. JW is funded by NIH/NEI P30 EY014104 and R01 EY022305. AH and DM are supported by an Australian National Health and Medical Research Council Practitioner Fellowship. EJ and $\mathrm{H}$ Choquet are supported by National Eye Institute (NEI) grant R01 EY027004, by the National Institute of Diabetes and Digestive and Kidney Diseases (NIDDK) grant R01 DK116738, and by the National Cancer Institute (NCI) grant R01 CA241623. The expression analysis was supported by the Australian National Health and Medical Research Council, the Joan and Peter Clemenger Foundation, and the Ophthalmic Research Institute of Australia. The funders had no role in study design, data collection and analysis, decision to publish, or preparation of the manuscript.

Competing interests: I have read the journal's policy and the authors of this manuscript have the following competing interests: APK is a paid consultant to Aerie, Allergan, Google Health, Novartis, Reichert, Santen and Thea. LRP is consultant for Verily, Eyenovia, Bausch+Lomb, and Nicox. JW is consultant for Allergan, Editas, Maze, Regenxbio and has a sponsored research grant from Aerpio.

\section{Abstract}

Optical Coherence Tomography (OCT) enables non-invasive imaging of the retina and is used to diagnose and manage ophthalmic diseases including glaucoma. We present the first large-scale genome-wide association study of inner retinal morphology using phenotypes derived from OCT images of 31,434 UK Biobank participants. We identify 46 loci associated with thickness of the retinal nerve fibre layer or ganglion cell inner plexiform layer. Only one of these loci has been associated with glaucoma, and despite its clear role as a biomarker for the disease, Mendelian randomisation does not support inner retinal thickness being on the same genetic causal pathway as glaucoma. We extracted overall retinal thickness at the fovea, representative of foveal hypoplasia, with which three of the 46 SNPs were associated. We additionally associate these three loci with visual acuity. In contrast to the Mendelian causes of severe foveal hypoplasia, our results suggest a spectrum of foveal hypoplasia, in part genetically determined, with consequences on visual function.

\section{Author summary}

The thickness of the inner retinal layers is one of the biomarkers for glaucoma, the leading cause of irreversible blindness globally. Here we utilised the large-scale of the UK Biobank and the images of the retina it contains to look for genetic variants that effect the thickness of the inner retina. We find many variants associated with this variable, but surprisingly only one that also affects glaucoma. Further analysis shows that glaucoma and genetically determined inner retinal thickness are not on the same genetic pathway and it is rather the change of thickness over time that is indicative of the disease. This is important as it invites the potential for the discovered variants to be used as a representation of baseline thickness in the clinic in the future. We also show that foveal hypoplasia, the lack of the normal valley-like shape of the central retina, is present at a population level in a mild form and is affected by three variants that also affect visual acuity. This is an interesting discovery as foveal hypoplasia was previously thought of as an outcome of rare Mendelian disease.

\section{Introduction}

The human retina is a highly structured tissue at the back of the eye which converts energy from photons focused by the cornea and lens into neuronal signals to provide vision. The retina is made up of distinct layers of cells, sometimes just one cell thick, which have specific functions in this signal processing. The inner retina, which is closest to the pupil of the eye, is responsible for the final stages of signal transmission through the eye before the signal exits via the optic nerve towards the brain (Fig 1A). Advances in optical coherence tomography (OCT) allow for non-invasive high-resolution imaging of retinal tissue structure, enabling the individual layers to be resolved. The central area of the retina, a region called the macula, has a distinct valley-like morphology that can be clearly seen using OCT. Here, the two inner retinal layers, the retinal nerve fibre layer (RNFL) and ganglion cell inner plexiform layer (GCIPL), taper to non-existence at the centre, the fovea, allowing less light scattering for incoming photons and so providing the region of highest acuity vision (Fig 1B) [1]. The morphology of the macula 

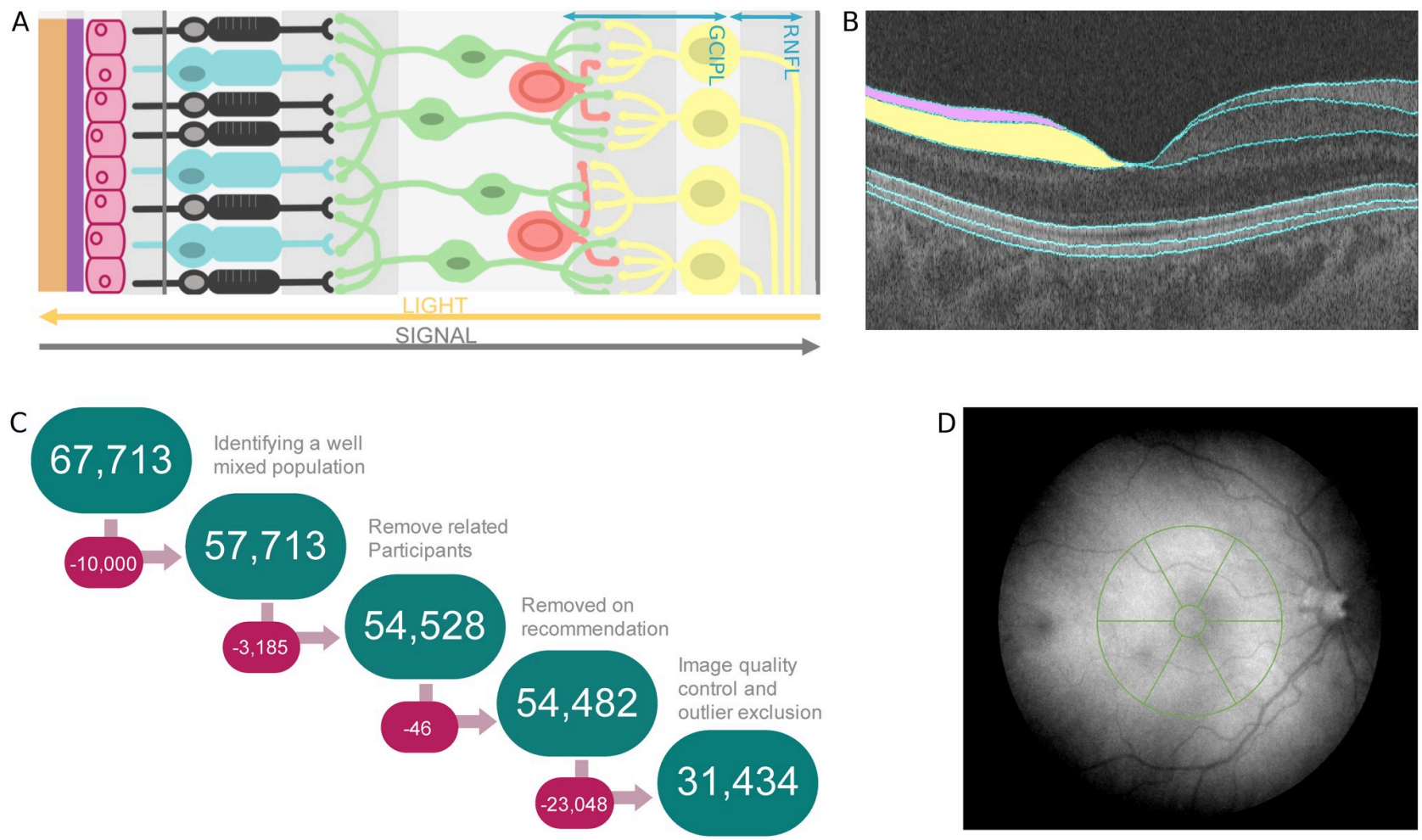

Fig 1. Retinal phenotype data and quality control. (A) A diagram illustrating the different retinal layers and the direction of travel for both the light stimulus and the neuronal signal. (B) An example of an Optical Coherence Tomography (OCT) image with segmented layer boundaries as labelled by the Topcon Advanced Boundary Segmentation (TABS) algorithm. On the left side of the image, the retinal nerve fibre layer (RNFL) is shaded pink, and the ganglion cell inner plexiform layer (GCIPL) is shaded yellow. (C) A schematic of the workflow applied during quality control, involving quality control of both the genotypic and phenotypic data. (D) A schematic of the Macula 6 grid, a commonly used partition matrix of the macular field when studying the inner retina. The matrix is comprised of 6 sections, with the central field being excluded from analysis.

https://doi.org/10.1371/journal.pgen.1009497.g001

has been studied previously, with variation in physiology associated with age $[2,3]$ and ethnicity $[4,5]$. There are also several diseases that exhibit changes in the thickness of the inner retina, such as diabetic retinopathy $[6,7]$, age-related macular degeneration $[8,9]$, multiple sclerosis [10, 11], Parkinson's disease [12] and other neurological disorders [13-16].

Most notably, glaucoma, the leading cause of irreversible blindness globally [17], causes thinning of the inner retinal layers [18]. The thickness of the Ganglion Cell Complex (GCC), the collective name for the RNFL and GCIPL, is one of the biomarkers used in diagnosis of primary open angle glaucoma (POAG) $[19,20]$. Studies have found genetic variants associated with glaucoma [21-24], as well as other endophenotypes of the disease such as intraocular pressure (IOP), one of the major risk factors for glaucoma [25, 26]. A number of well-defined loci are known to affect POAG, with over 100 loci reportedly associated with the disease, including at CDKN2B-AS1, SIX1/SIX6, CAV1/CAV2, TMCO1 and GAS7 amongst others [22, $24,27]$. Genetic influence on retinal morphology has been previously studied [28] but largely in smaller cohorts or focused on looking at the retina as a whole [29].

In this study we utilised the large UK Biobank resource where in the latter stages of systematic phenotyping, 67,321 volunteers had OCT imaging centred on the macula [30]. The UK Biobank is a large, well-studied prospective cohort in the UK, with recruitment of adults displaying good general health. On recruitment, participants had a large number of physiological, health and lifestyle-related variables measured. They also consented to on-going linkage of their medical records. The entire $\sim 500,000$ person cohort has been genotyped and imputed 
[31]. This dataset therefore provides a well-powered resource to produce a comprehensive view of retinal morphology genetics. In this study we have focused on the morphology of the inner retina, comprising the RNFL and GCIPL, with particular focus on related diseases of these layers, such as POAG. We find 46 loci associated with variation in mean thickness of at least one of these two layers. Many of these loci are associated with other eye traits and broader anthropometric and neurodevelopmental traits. Further interrogation of these SNPs reveals a subset are also associated with a measure representative of foveal hypolasia, clinically defined as the absence of a depression at the fovea and continuation of the inner retinal layers into the foveal area [32]. The same SNPs are associated with pigmentation and visual acuity. Interestingly, the majority of discovered loci do not coincide with the extensive glaucoma genetic datasets. We show using Mendelian randomisation that IOP, a well-known risk factor for POAG and target of POAG therapy, has strong support for being on the causal pathway of POAG. However genetic variation in inner retinal thickness does not have strong support for a causal relationship with either POAG or IOP. The established use of retinal thickness as a biomarker for POAG is consistent with a change from baseline due to the progression of the disease; however, genetically determined inner retinal thickness largely does not impact development of POAG.

\section{Results}

We performed standard OCT image and genetic quality control to define a high quality and genetically well-mixed subset of the imaged UK Biobank population (see Methods). This resulted in 31,434 people who passed both imaging and genotype filters (Fig 1C). This subset of people had similar sex and age profiles to the overall population (S1 Table).

We then performed genome-wide association studies (GWAS) of the mean thickness across the Macula 6 grid of the two inner retinal layers, the RNFL and GCIPL, averaged across left and right eyes (Fig 1D). Both GWAS identified many significant loci $\left(\mathrm{P}<5 \times 10^{-8}\right)$ and showed minimal evidence of inflation due to residual population structure (RNFL: $\lambda \mathrm{GC}=1.11$, Linkage Disequilibrium Score regression $($ LDSC) Intercept $=1.01$, Ratio $=0.05$; GCIPL: $\lambda$ $\mathrm{GC}=1.12$, LDSC Intercept $=1.01$, Ratio $=0.05$, where ratio $=($ LDSC intercept -1$) /\left(\right.$ mean $\left(\chi^{2}\right)$ 1)), characteristic of a robust GWAS result (S1 Fig). Given that several of the significant loci were common between the two studies, we combined the two GWAS using meta-analysis methods implemented in MTAG [33] (Fig 2). We also explored performing GWAS of the separate thickness measures for each of the Macula 6 subfields, or of principle components derived from the Macula 6 grid subfield measurements. Due to the large sample size of this study there was little difference in the discovery power when using the high dimensional phenotypes compared to the simpler two-phenotype method. We favoured the simpler two-phenotype model which has fewer parameter choices and is also in-keeping with common clinical usage of these measures.

There are 46 lead loci discovered across the two phenotypes, listed in Table 1 (additional information available in S2 Table). Many of these loci lie within or near genes which harbour mutations causing a variety of eye phenotypes. These include rs1800407 and rs1042602, at loci previously associated with oculocutaneous albinism (in OCA2 and TYR respectively) [34]. There are also multiple loci that are in or near genes associated with refractive error (TSPAN10, GNB3, SNAP91, COBL). Several of the significant loci overlapped with those previously associated with overall retinal thickness [29]. However 36 of our variants are novel and have not previously been associated with retinal morphology. Surprisingly, there was only one locus, rs1254276 at SIX6, that was previously associated with POAG [27] (S3 Table), though there were several loci that were previously associated with IOP (TYR, PIK3C2A, NSF, 


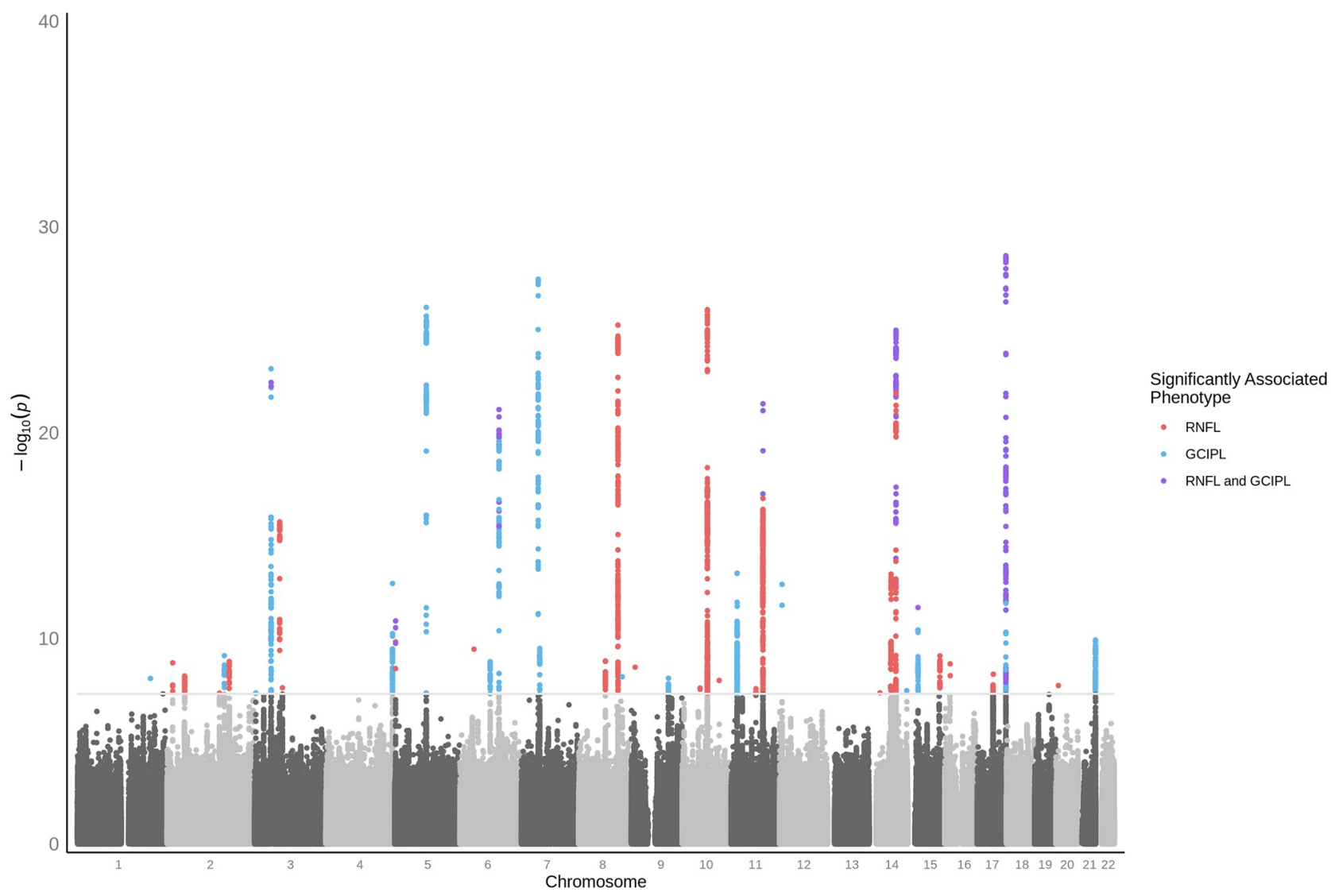

Fig 2. Genome-wide association study of inner retinal thickness phenotypes. Manhattan plot of inner retinal thickness phenotype GWAS p-values, resulting from meta-analysis across RNFL and GCIPL. Variants significantly associated $\left(\mathrm{P}<5 \times 10^{-8}\right)$ with only RNFL are highlighted in red, those significantly associated with only GCIPL are highlighted in blue, and those significantly associated with both inner retinal layers are highlighted purple.

https://doi.org/10.1371/journal.pgen.1009497.g002

TSPAN10, STOX2) [26]. Replication was sought in two independent datasets. Replication in the Rotterdam study dataset (S4 Table) saw correlation of the effect sizes of meta-analysed loci associated with RNFL and GCIPL (Pearson's $\mathrm{R}=0.74, \mathrm{P}=1.25 \times 10^{-6}$ ). In the Raine dataset (S5 Table), correlation of effect sizes is seen for loci associated with the GCIPL (Pearson's $\mathrm{R}=0.84, \mathrm{P}=5.95 \times 10^{-6}$ ), however not in RNFL (Pearson's $\mathrm{R}=-0.03, \mathrm{P}=0.88$ ). The smaller sample size of both replication data sets meant that many loci would not pass genome-wide significance. The younger age of individuals within the Raine data set may account for the weaker replication. Despite the lack of replication on RNFL in the Raine study, we believe the correlation between GCIPL and RNFL in this study and the association with other retina associated phenotypes indicate that the RNFL results are robust.

We investigated whether the SNPs within our discovered SNP set had sex-specific effects by applying a linear model of RNFL and GCIPL thickness that included an interaction term between the discovered SNPs and genetically determined sex. The interaction was not statistically significant following Bonferroni correction for any of the SNPs affecting GCIPL thickness, but five SNPs had a statistically significant sex-specific effect on RNFL thickness. For example, rs146652416 at FOXG1 had a significant sex interaction effect $\left(\mathrm{P}=8.08 \times 10^{-4}\right.$, S2 Fig). FOXG1 encodes a fork-head transcription factor involved in brain development [35]. However all of these SNPs had a homozygous alternative allele frequency of less than 15 when stratified by sex. Therefore, whilst these results suggest that sex might impact some of the 
Table 1. 46 SNPs associated with GCIPL or RNFL thickness and annotations of ocular and general biology phenotypes. Variants considered to be representative of a single locus, examples of allelic heterogeneity, are highlighted in the same colour alternating white and grey. For full results including effect size, effect allele specification and standard error please see S2 Table.

\begin{tabular}{|c|c|c|c|c|c|}
\hline SNP & Chr & P-value & Nearest gene & Ocular Phenotypes & Non-ocular Phenotypes \\
\hline rs72739513 & 1 & 8.88E-09 & ADORA1 & & \\
\hline rs79833181 & 2 & $1.55 \mathrm{E}-09$ & NBAS & & \\
\hline rs13010692 & 2 & $6.72 \mathrm{E}-09$ & STON1-GTF2A1L & & \\
\hline rs980772 & 2 & $4.62 \mathrm{E}-08$ & TEX41 & & $\begin{array}{l}\text { Eosinophil count, Lymphocytes, Neutrophil count, Smoking, White } \\
\text { blood cell count }\end{array}$ \\
\hline rs12998032 & 2 & $6.97 \mathrm{E}-10$ & CCDC148 & & \\
\hline rs2271758 & 2 & $1.34 \mathrm{E}-09$ & SLC25A12 & & $\begin{array}{l}\text { Age completed full time education, Anthropometric traits, Reaction } \\
\text { time }\end{array}$ \\
\hline rs13083522 & 3 & $4.51 \mathrm{E}-08$ & $C R B N$ & & \\
\hline rs 17279437 & 3 & $7.81 \mathrm{E}-24$ & SLC6A20 & Macular thickness & $\begin{array}{l}\text { Blood metabolite levels, BMI, Hyperglycinuria, Iminoglycinuria, } \\
\text { Overall health rating, Urinary metabolites }\end{array}$ \\
\hline rs62252355 & 3 & $2.17 \mathrm{E}-16$ & FRMD4B & & \\
\hline rs149831820 & 3 & $2.53 \mathrm{E}-08$ & $\mathrm{ROBO} 2$ & & \\
\hline rs66511946 & 4 & $2.15 \mathrm{E}-13$ & STOX2 & & \\
\hline rs2004187 & 5 & $1.43 \mathrm{E}-11$ & $\operatorname{IRX} 2$ & Macular thickness & Acute renal failure \\
\hline rs17421627 & 5 & $8.09 \mathrm{E}-27$ & LINC00461 & $\begin{array}{l}\text { Retinal vascular calibre, Macular } \\
\text { thickness }\end{array}$ & Seen doctor for nerves/anxiety \\
\hline rs527871768 & 6 & 3.37E-10 & KIF6 & & \\
\hline rs13215351 & 6 & $1.36 \mathrm{E}-09$ & SNAP91 & Spherical power & Bronchiectasis, Menarche, Napping, Standing height \\
\hline rs9398171 & 6 & $7.51 \mathrm{E}-22$ & FOXO3 & Macular thickness & $\begin{array}{l}\text { Anthropometric traits, BMI, Coffee intake, Fat-free mass, Intelligence, } \\
\text { Lung function, Menarche, Schizophrenia }\end{array}$ \\
\hline rs11762530 & 7 & $3.45 \mathrm{E}-28$ & IGFBP3 & & Body Mass \\
\hline rs73348111 & 7 & $7.15 \mathrm{E}-10$ & $I K Z F 1$ & & Mean reticulocyte volume, Monocyte percentage \\
\hline rs35001871 & 7 & 1.17E-09 & GRB10 & & \\
\hline rs12719025 & 7 & $3.09 \mathrm{E}-10$ & $C O B L$ & $\begin{array}{l}\text { Macular thickness, Spherical power, } \\
\text { Strong/weak meridian }\end{array}$ & \\
\hline rs6989495 & 8 & $1.27 \mathrm{E}-09$ & RDH10 & & \\
\hline rs115520750 & 8 & $2.54 \mathrm{E}-10$ & ANGPT1 & & \\
\hline rs13271359 & 8 & $5.89 \mathrm{E}-26$ & RSPO2 & & \\
\hline rs376067714 & 8 & $1.28 \mathrm{E}-18$ & RSPO2 & & \\
\hline rs4871827 & 8 & 7.41E-09 & DEPTOR & & Asthma, Height, Heel bone mineral density, Platelet count \\
\hline rs118031671 & 9 & $2.53 \mathrm{E}-09$ & PTPRD & & \\
\hline rs2787394 & 9 & $8.64 \mathrm{E}-09$ & INVS & Macular thickness & BMI, Body Mass, Weight \\
\hline rs1947075 & 10 & $2.60 \mathrm{E}-08$ & ARHGAP22 & Macular thickness & \\
\hline rs10762201 & 10 & $1.05 \mathrm{E}-26$ & ATOH7 & & Anthropometric traits \\
\hline rs181211282 & 10 & $1.12 \mathrm{E}-08$ & MRPL43 & & \\
\hline rs2008905 & 11 & $6.81 \mathrm{E}-14$ & PIK3C2A & & Platelet count, Schizophrenia, Standing height \\
\hline rs12574166 & 11 & $2.82 \mathrm{E}-08$ & LINC02747 & & Breast cancer \\
\hline rs1042602 & 11 & $3.96 \mathrm{E}-22$ & TYR & $\begin{array}{l}\text { Eye colour, IOP, Macular thickness, } \\
\text { Oculocutaneous albinism }\end{array}$ & $\begin{array}{l}\text { Depression, Hair colour, Heel bone mineral density, Nerves, Skin } \\
\text { pigmentation, Tanning }\end{array}$ \\
\hline rs5442 & 12 & $2.36 \mathrm{E}-13$ & GNB3 & $\begin{array}{l}\text { Hypermetropia, Macular thickness, } \\
\text { Myopia, Spherical power }\end{array}$ & \\
\hline rs146652416 & 14 & $4.46 \mathrm{E}-08$ & FOXG1 & & \\
\hline rs17095953 & 14 & $1.76 \mathrm{E}-10$ & $D A A M 1$ & & \\
\hline rs1254276 & 14 & $7.52 \mathrm{E}-14$ & SIX6 & $\begin{array}{l}\text { Age started wearing glasses, Primary open } \\
\text { angle glacuoma (POAG) }\end{array}$ & Anthropometric traits, Heel bone mineral density, Menarche \\
\hline rs10140252 & 14 & $1.05 \mathrm{E}-25$ & BBOF1 & & $\begin{array}{l}\text { Mean corpuscular haemoglobin, Mean corpuscular volume, Red blood } \\
\text { cell count, Red cell distribution width }\end{array}$ \\
\hline
\end{tabular}

(Continued) 
Table 1. (Continued)

\begin{tabular}{|c|c|c|c|c|c|}
\hline SNP & Chr & P-value & Nearest gene & Ocular Phenotypes & Non-ocular Phenotypes \\
\hline rs35337422 & 14 & $3.50 \mathrm{E}-08$ & TDRD9 & Myopia, Spherical equivalent & \\
\hline rs1800407 & 15 & $3.19 \mathrm{E}-12$ & OCA2 & $\begin{array}{l}\text { Age started wearing glasses, Cataract, Eye } \\
\text { colour, Oculocutaneous albinism }\end{array}$ & Hair colour, Skin colour, Tanning response \\
\hline rs1470108 & 15 & $7.09 \mathrm{E}-10$ & $A E N$ & & Standing height \\
\hline rs117304899 & 16 & $1.74 \mathrm{E}-09$ & & & \\
\hline rs117300236 & 17 & 5.57E-09 & NSF & & $\begin{array}{l}\text { Balding, Forced expiratory volume, Haemoglobin concentration, } \\
\text { Height, Mean corpuscular volume, Mean sphered cell volume, } \\
\text { Neuroticism, Neutrophil percentage, Red blood cell count, Sensitivity }\end{array}$ \\
\hline rs7503894 & 17 & $2.49 \mathrm{E}-29$ & TSPAN10 & $\begin{array}{l}\text { Age started wearing glasses, Astigmatism, } \\
\text { Cataract, Cylindrical power, Spherical } \\
\text { power }\end{array}$ & Hair colour, Tanning \\
\hline rs143330165 & 20 & $1.97 \mathrm{E}-08$ & LINC01428 & & \\
\hline rs7277632 & 21 & $1.20 \mathrm{E}-10$ & РСВР3 & & \\
\hline
\end{tabular}

phenotypes, the results cannot be considered robust and larger sample sizes would be needed to explore this in more detail.

In addition to the TYR, OCA2 and TSPAN10 loci, which we discuss in more depth below, many of the loci associated in this study are close to well established genes involved in other aspects of ocular biology. Associated loci include; rs10762201 and rs2004187 near the ATOH7 and $I R X 2$ genes respectively, both associated with eye development [36, 37]; rs79833181 near NBAS associated with optic atrophy [38]; rs149831820 near ROBO2 associated with retinal ganglion cell axon guidance [39, 40]; and rs73348111 (at IKZF1), rs13271359 and rs376067714 (both at RSPO2) involved in differentiation and retinal cell definition [41, 42]. These SNPs and the others in Table 1 show the link between common variation and the large effects of either Mendelian disease or animal models in eye biology, and provide both feasible paths for more exploration of the biology and the impact of natural population variation on specific aspects of eye biology.

Several of the loci we identified were previously associated with refractive error, despite us adjusting for refractive error in our statistical models. However, two of the most established loci associated with refractive error, at LAMA2 (rs12193446) [43] and GJD2 (rs524952) [44], were not significantly associated with inner retinal morphology in our MTAG analysis $(\mathrm{P}=0.32$ and $\mathrm{P}=0.12$, respectively). This suggests that there are some shared genetic processes between myopia and inner retinal morphology and that these results are not being driven by residual confounding or magnification artefact due to refractive error.

The presence of loci associated with oculocutaneous albinism, whose effect on foveal morphology has been well documented [45], prompted us to examine an additional measure of retinal morphology. We created a simple model of the total retinal thickness across the macula from the raw OCT images (see Methods), and saw how such models varied when stratified by the genotype at each of the 46 lead loci. This revealed that some variants have a more notable diffuse effect across the whole scanned retinal area, while others have a predominant effect on the fovea (Fig 3 and S3 Fig). Here we describe the foveal-centred changes in thickness as mild foveal hypoplasia. To produce a statistical value for this difference, a linear model was constructed modelling the effect of each of the discovery set loci on the total thickness of the retina at the central fovea. Three SNPs showed significant differences in retinal thickness at the central fovea: TSPAN10 (rs7503894, P $\left.=1.00 \times 10^{-25}\right)$, TYR $\left(\mathrm{rs} 1042602, \mathrm{P}=1.23 \times 10^{-16}\right)$ and OCA2 $\left(\mathrm{rs} 1800407, \mathrm{P}=2.61 \times 10^{-10}\right)$. 

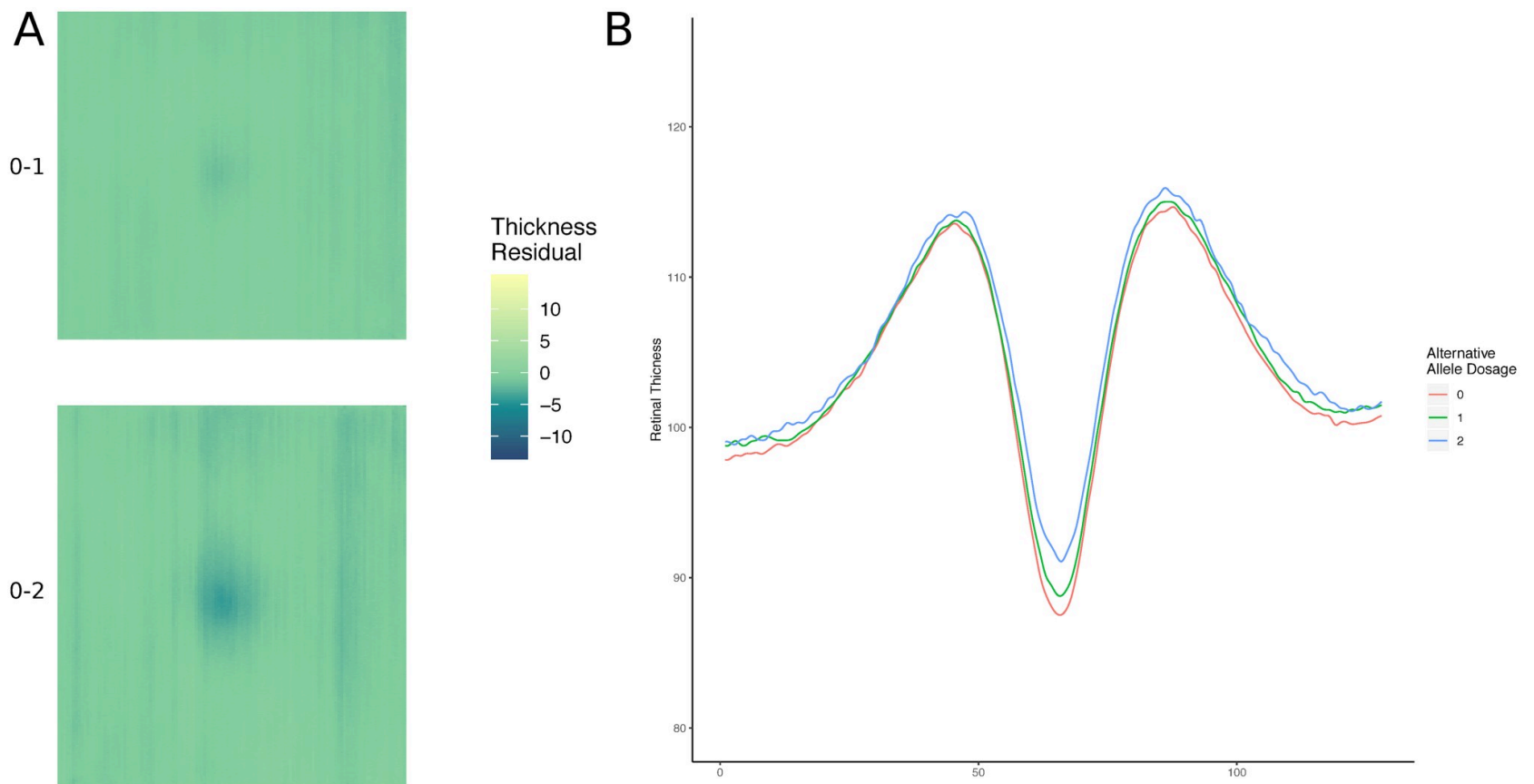

Fig 3. Higher dimensional detailed retinal phenotyping. (A) A model of the macular field showing the difference in mean total retinal thickness between those with homozygous reference and heterozygous alleles (top), and homozygous reference and homozygous alternative alleles (bottom) at rs1042602 (TYR). (B) Models of the mean overall retinal thickness across three groups defined by their allele state, homozygous reference (0), heterozygous (1) or homozygous alternative (2) at rs1042602 (TYR). The y axis represents total retinal thickness.

https://doi.org/10.1371/journal.pgen.1009497.g003

Due to the pre-established link of these SNPs with pigmentation, the effect of the three foveal thickness-associated SNPs on both hair colour and skin colour was explored using a linear model. All three loci were significantly associated with hair colour (TSPAN10: $\mathrm{P}=1.27 \mathrm{x}$ $10^{-9}$, TYR: $\mathrm{P}=5.08 \times 10^{-12}$, OCA2: $\mathrm{P}=4.82 \times 10^{-23}$, Fig 4). The direction of effect of hair colour change (ranking hair colour from light to dark; see Methods) relative to the direction of effect on foveal hypoplasia was inconsistent across the loci. For the TYR and TSPAN10 loci, the allele associated with greater foveal hypoplasia was also associated with lighter hair colour. However, for the OCA2 locus, the allele associated with greater foveal hypoplasia was associated with darker hair colour. Only the well-described oculocutaneous albinism variants, TYR and OCA2, were significantly associated with skin colour $\left(\mathrm{P}=2.19 \times 10^{-4} \& 2.31 \times 10^{-3}\right.$, respectively). There are only 19 individuals in UK Biobank with documented albinism from the Hospital Episode Statistics (ICD10 codes); this is likely an underestimate due to under-reporting and diagnosis, but the frequency of the risk variants at these loci are far higher than the documented levels of oculocutaneous albinism in the UK population, suggesting that the majority of these people are not clinically classified as having eye defects due to the condition. These results show that common natural variation in pigmentation pathways influence retinal development across broad populations but with complex, pleiotropic effects (see Discussion).

To determine whether any of the retinal morphology-associated variants additionally had effects on retinal function, we examined their association with visual acuity (S6 Table). Three of the 46 variants were significantly associated with visual acuity at a Bonferroni-corrected threshold $\left(\mathrm{P}=3.54 \times 10^{-19}, 3.61 \times 10^{-8}\right.$ and $2.16 \times 10^{-5}$ for variants at TSPAN10, TYR and OCA2 respectively). Interestingly, these three loci are also the loci associated with the foveal hypoplasia phenotype. The relationship between foveal hypoplasia and visual acuity had a consistent 


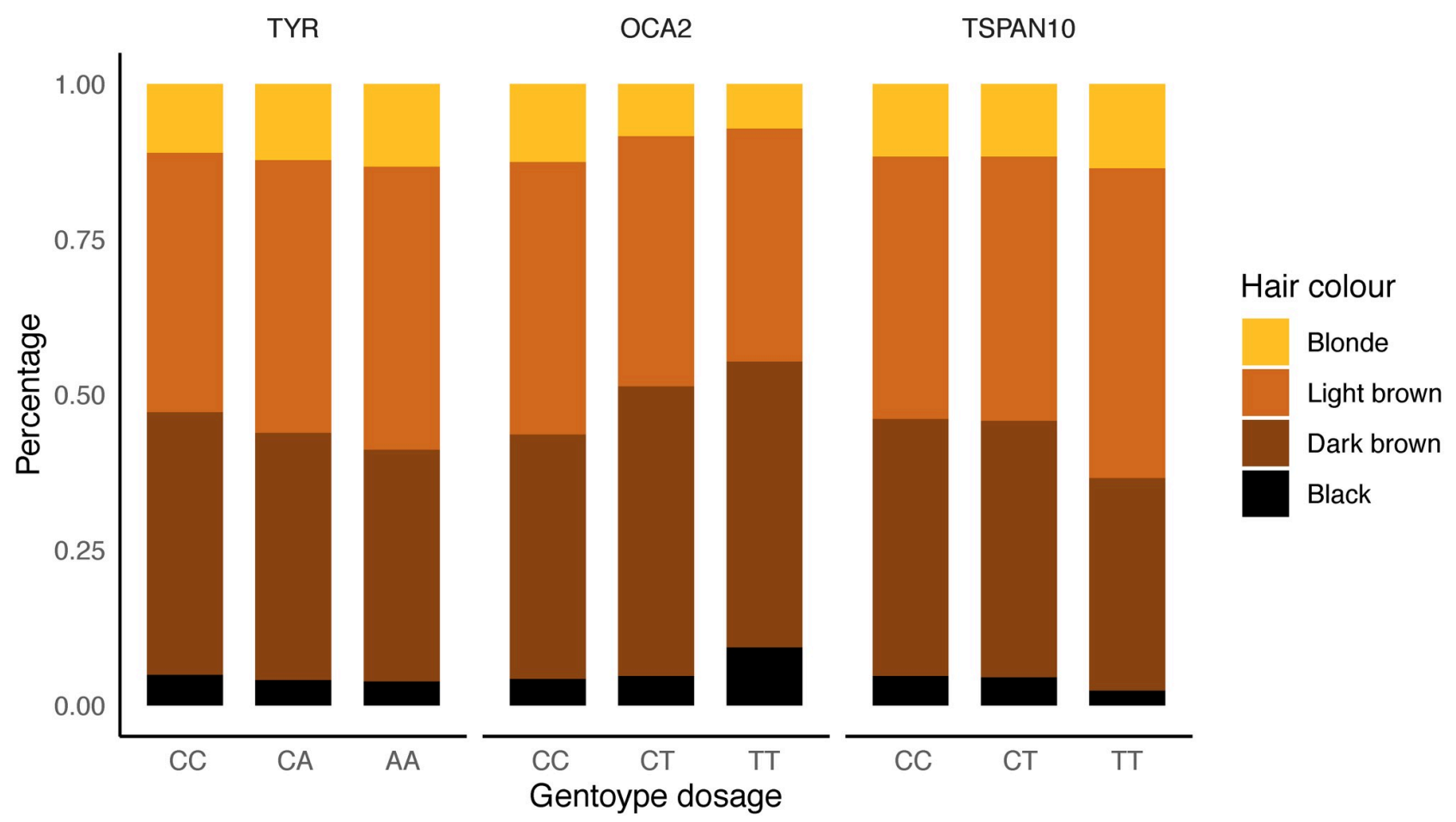

Fig 4. Hair colour stratified by genotype state. Proportions of self-reported hair colour within our dataset population plotted stratified by genotype at the three overall retinal thickness associated loci. Genotypes are aligned so the allele on the right cause a thicker foveola. From left to right: TYR (rs1042602), OCA2 (rs1800407), TSPAN10 (rs7503894).

https://doi.org/10.1371/journal.pgen.1009497.g004

direction across the three variants; the allele associated with a greater degree of foveal hypoplasia was associated with worse visual acuity.

To further explore the underlying biological mechanisms and pathways associated with the traits, we used GARFIELD [46] that associates the full spectrum of GWAS loci with regulatory features from different cell or tissue types. There is an over 10-fold enrichment for loci associated with either RNFL or GCIPL $(\mathrm{P}<0.05)$ in eye tissues (Fig 5). Similar-fold enrichment is seen in pancreas $(>15 \mathrm{x})$, kidney $(>10 \mathrm{x})$, blood $(>15 \mathrm{x})$ and brain $(>15 \mathrm{x})$ (See Discussion).

Additionally, we used DEPICT [47] to identify enriched pathways and tissues from GWAS summary statistics. No gene sets reached significance following false discovery rate (FDR) correction, however several reached nominal significance $(\mathrm{P}<0.05, \mathrm{~S} 7 \mathrm{Table})$. These included ocular associated gene sets such as Cellular response to light stimulus $\left(\mathrm{P}=3.60 \times 10^{-4}\right)$. Interestingly, there were a number of gene sets involved in insulin that were nominally enriched for within our list of discovered loci. These gene sets include Reactome signalling by insulin receptor and Cellular response to insulin stimulus. The eye was the tissue in which our identified loci were enriched with the highest nominal significance $(\mathrm{P}<0.05)$, however this did not reach significance following FDR adjustment (S8 Table).

We sought to characterise the expression profile of genes at loci associated with variation in inner retinal thickness across induced pluripotent stem cell-derived retinal organoids. Overall, there was increased expression of many of the genes at our discovered loci in the different retinal cell types (S4 Fig). Several of the genes have high levels of expression in the retinal ganglion cells, the predominant cell type within the inner retina. These include PCBP3, CRBN, DAAM1, $P T P R D, N S F, F O X O 3$ and IRX2 amongst others. In contrast, some of the genes showed low expression in the retinal ganglion cells but distinct patterns of expression in other cell types. 


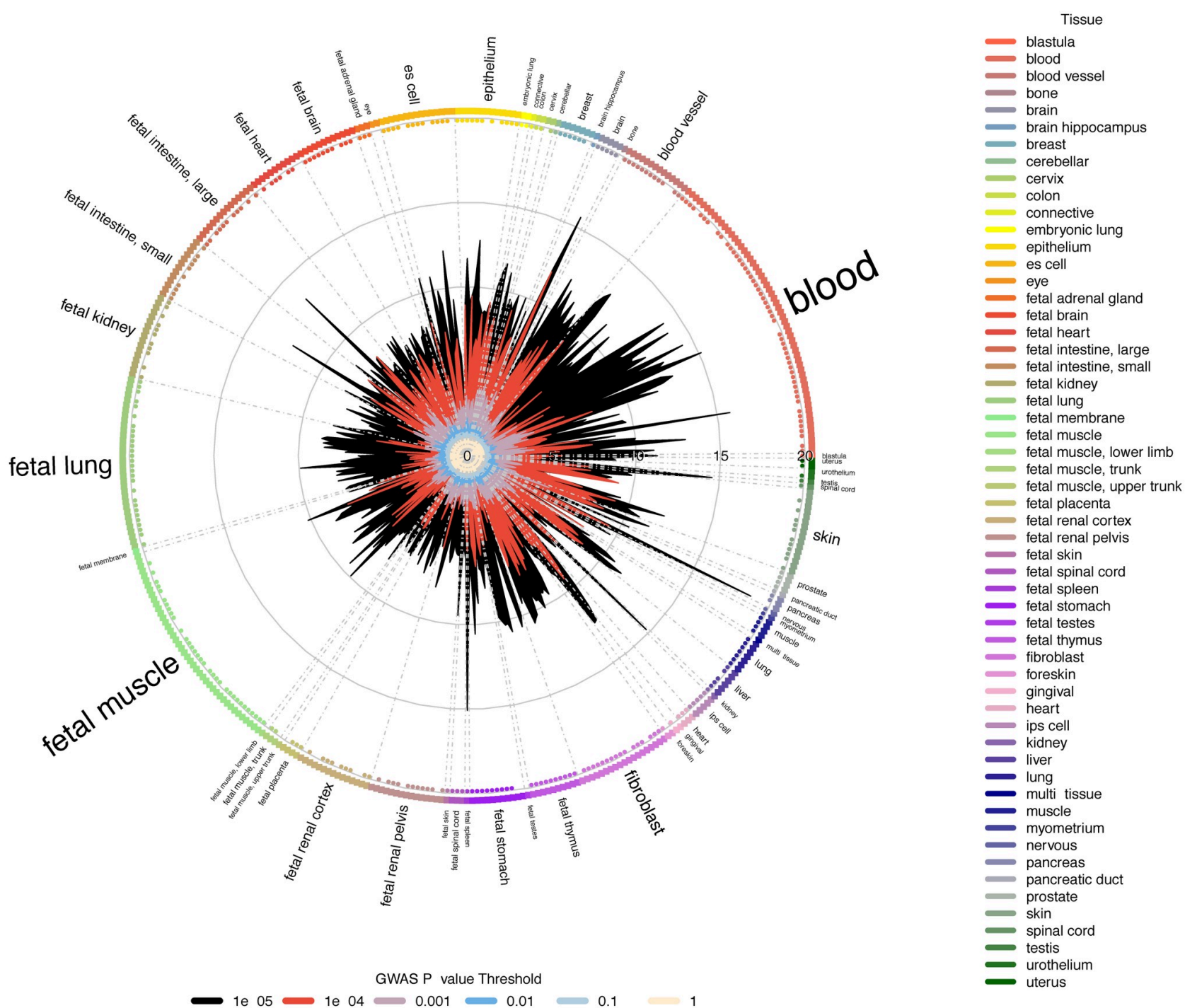

Fig 5. Regulatory feature association using GARFIELD. Wheel plot of enrichment analysis on meta-analysed GCIPL and RNFL GWAS results across a number of cell types, as performed in GARFIELD. Associations at different GWAS P-value thresholds are represented in different colours.

https://doi.org/10.1371/journal.pgen.1009497.g005

For example CCDC176 has increased expression in retinal pigment epithelial cells. Many of the genes had high levels of expression in retinal progenitor cells, which can later differentiate into ganglion cells, as well as other types of retinal neurons.

Prompted by the surprising lack of overlap between genetic variants associated with the inner retinal phenotypes and the established inner retinal disease of glaucoma, we performed two-sample Mendelian randomisation studies between the retinal morphology traits (RNFL and GCIPL), IOP and POAG. Mendelian randomisation is a statistical technique that uses genetics to test a suspected causal relationship between an "exposure" variable (in this case IOP, RNFL or GCIPL thickness) and an outcome variable (in this case POAG or IOP). For POAG, summary statistics were taken from the International Glaucoma Genetics Consortium (IGGC) meta-analysis [24], and for IOP, summary statistics taken from [26]. As expected, there was evidence for a strong causal link between IOP and POAG, with strong concordance of the effects of variants on IOP with the effects on POAG (Fig 6A and 6B). This concordance 
A
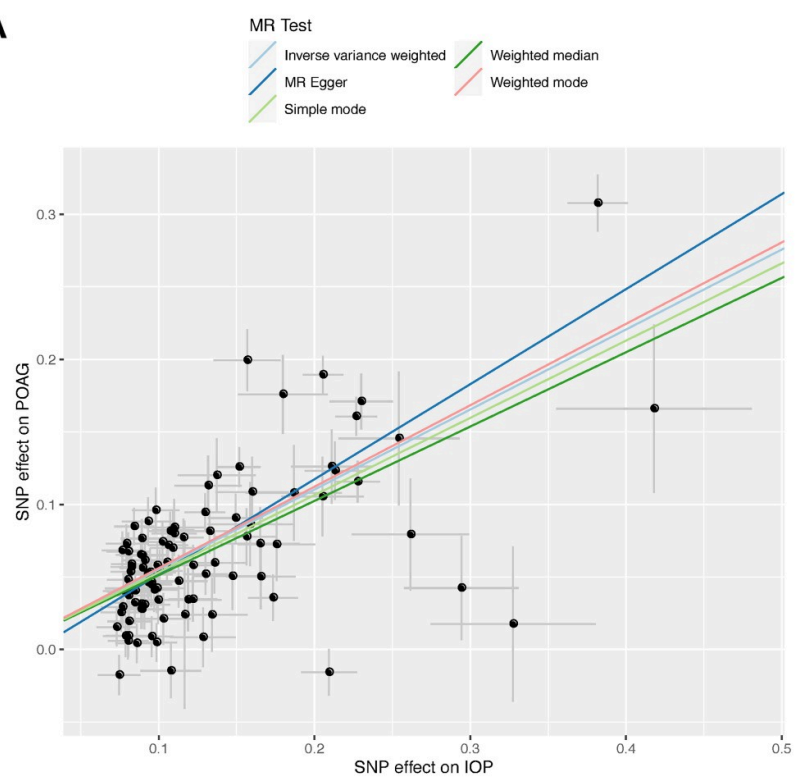

C

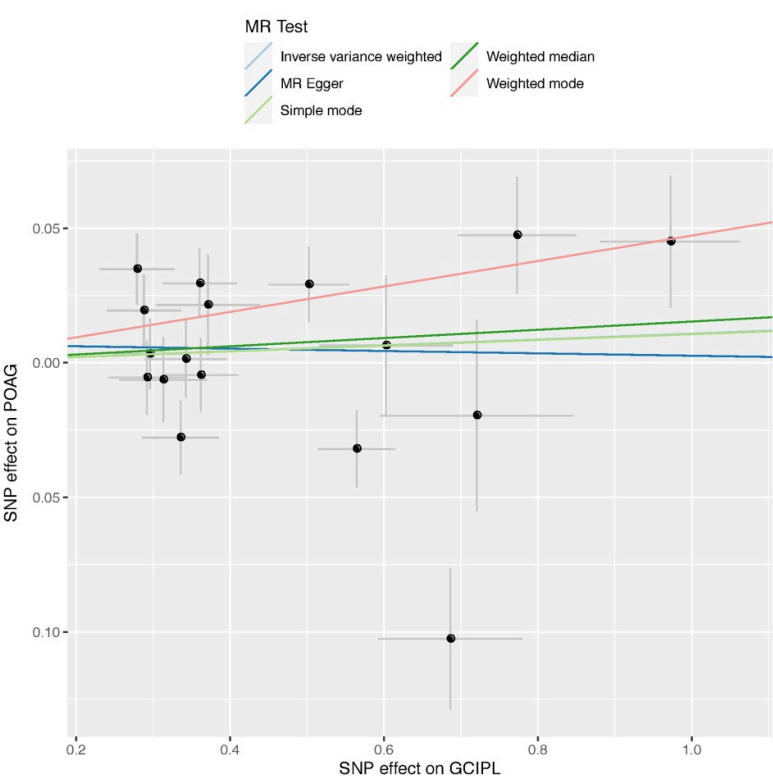

B

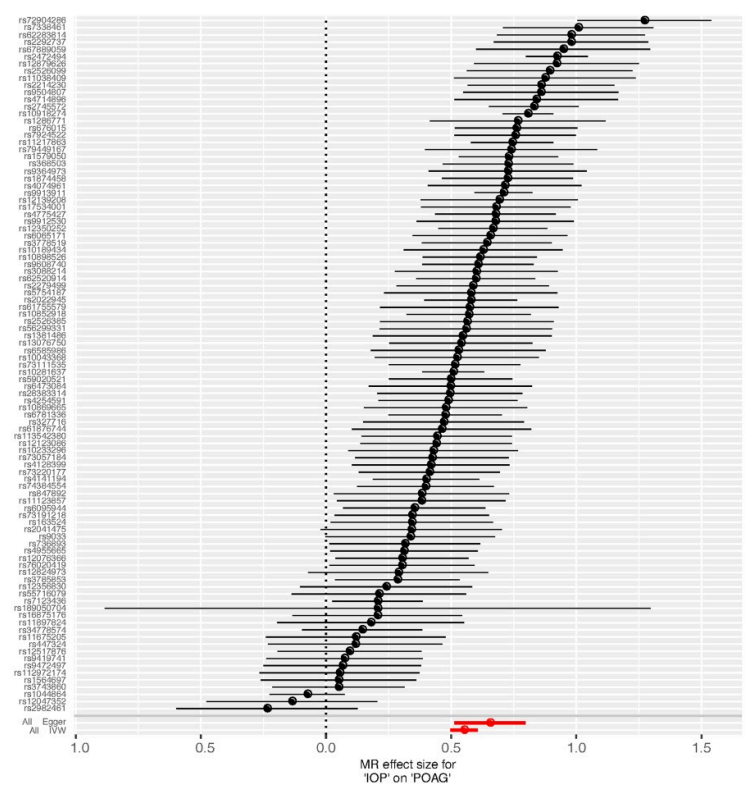

D

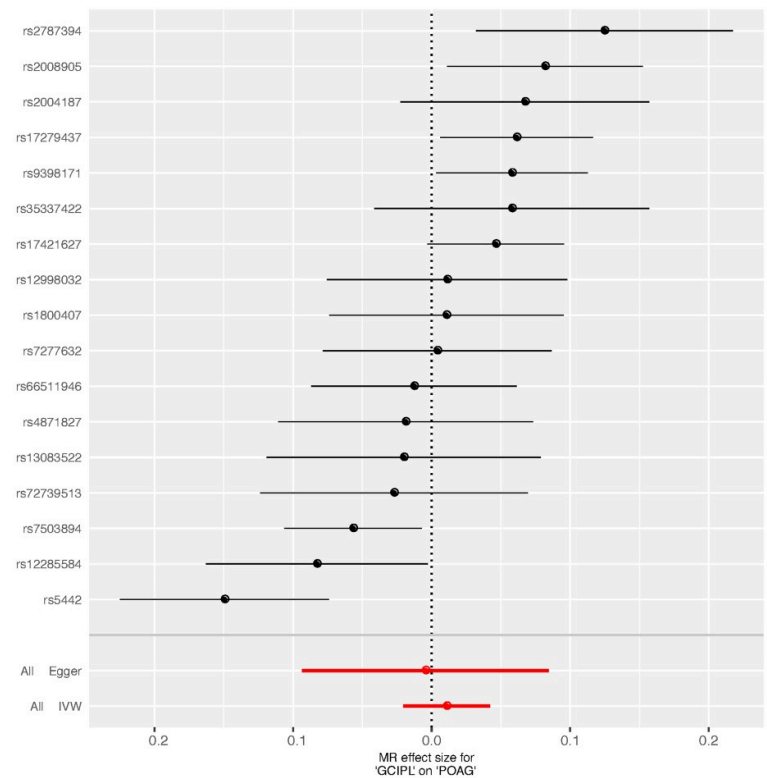

Fig 6. Mendelian randomisation analysis. (A) Scatter plot of the relationship between the effect size of SNPs found significantly associated to intraocular pressure (IOP), and the effect of those SNPs on primary open angle glaucoma (POAG). (B) Forest plot showing effect size and direction of SNPs significantly associated with IOP on POAG. (C) Scatter plot of the relationship between the effect size of SNPs found significantly associated with ganglion cell inner plexiform layer (GCIPL) thickness, and the effect of those SNPs on POAG. (D) Forest plot showing effect size and direction of SNPs significantly associated with the thickness of the GCIPL on POAG. POAG summary statistics were taken from the POAG International Glaucoma Genetics Consortium (IGGC) meta-analysis [24]. Summary statistics for genetic association studies of IOP, were taken from [26].

https://doi.org/10.1371/journal.pgen.1009497.g006

is consistent with evidence that lowering IOP reduces the risk of the progression of POAG [48]. In contrast, there was no evidence of a causal effect of either GCIPL or RNFL on IOP or POAG (Fig 6C and 6D and S5 and S6 Figs). Conducting the analysis with POAG or IOP as the exposure, and retinal thickness as the outcome, there was no evidence for an effect of IOP on retinal layer thickness, and at most weak support via one meta-analysis technique for a causal 
relationship between POAG and retinal thickness of both GCIPL and RNFL (S7 and S8 Figs and S9 Table); given the strong causal link of IOP to POAG shown by both MR and drug treatments, if this causal link between POAG and inner retinal thickness is present, this data indicates that it is not on the causal pathway with IOP, and likely takes a different biological route.

The lack of concordance between the genetically determined thickness of RNFL or GCIPL and POAG is in contrast to their established use as diagnostic biomarkers for POAG [49, 50]. Consistent with previous epidemiological studies, the UK Biobank datasets shows thinner GCC for diagnosed glaucoma patients (S9 Fig). The SIX6 locus is the only locus clearly associated with GCIPL or RNFL and glaucoma. The same locus is also associated with a number of developmental traits, such as age of menarche and anthropometric traits. The role of the SIX6 locus in development of the neural retina has been demonstrated in a zebrafish model, and a common missense variant Asn141His (rs33912345) has been implicated as causal [51].

\section{Discussion}

We have performed the first large-scale genetic association study on inner retinal morphology. We explored a variety of options of how to model the phenotype, but with the large sample size present in UK Biobank, there was little difference in discovery power between a high dimensional perspective compared to the more straightforward GWAS of the measurements used in clinical practice, namely mean RNFL and GCIPL thickness across the macular field and across left and right eyes. We robustly discovered 46 loci associated with at least one of the inner retinal thickness phenotypes across the genome. Many of the discovered loci are related to eye phenotypes; a notable association was to variants in genes associated with oculocutaneous albinism, which is known to affect the retinal pigment epithelium in the outer retina in addition to causing foveal hypoplasia [52]. Further exploration of these and one other locus shows that some minor foveal hypoplasia occurs in many individuals due to these variants. Although all three variants were associated with hair colour, the direction of effect on hair colour is not consistent with the direction of effect of foveal hypoplasia, showing that there is a complex relationship between the pigmentation pathway in retinal development compared to hair colour. The TSPAN10 locus is less well described as being involved with pigmentation, with some evidence that it is involved in eye pigmentation specifically [53]. Ideally association to eye colour would also be tested, however the UK Biobank does not currently contain such phenotypic information. Although there is a complex genetic architecture for these three loci, it is clear that they collectively impact foveal development. Notably the three associated loci also showed significant association with visual acuity, implying a link between even subtle foveal hypoplasia and visual function; to our knowledge this is the first time genetic variation has been associated with visual acuity, as measured using a LogMAR chart. As the internal segmentation of the layers and the outline of the fovea were not available from the Topcon Advanced Boundary Segmentation (TABS) algorithm [54], the OCT segmentation software, it is complex to deconstruct which aspect of macular structure is changing the layer thickness measurements for these loci. The simplest explanation for these loci's association is that the mild foveal hypoplasia caused by these variants is systematically changing the average GCIPL and RNFL measurements, potentially due to incorrect positioning of the macula within the Macula 6 grid during scan acquisition. This suggests there is room for possible improvements in the image processing and measurement derivation. As these measurements are often used in clinical practice, in particular in the diagnosis of POAG, this retinal developmental variation will confound some uses of the current measurement schemes both in research and clinically.

The GWAS loci are also associated with a variety of other traits, with many loci also being associated with anthropometric traits, asthma, blood cell related traits, and some neurological 
traits. This points to the broad and complex pleiotropy across biology but also suggests an opportunity for using the optically accessible retinal tissue, part of the central nervous system, as a source of potential biomarkers for other diseases $[55,56]$. Consistent with this broad pleiotropy, GWAS loci are enriched in DNaseI hypersensitive sites in pancreas, kidney, blood and brain cells. The overlap with kidney tissue is interesting given the longstanding association between kidney and retinal disease [57-59]. This tissue overlap is also consistent with loci including rs2004187 (IRX2), which is associated with renal failure. The increased expression of the genes labelled by our discovered loci in retinal progenitor cells highlights the role of development in retinal morphology.

A surprise in our analysis was a consistent lack of overlap of our discovered loci with POAG loci, which has a large-scale consortium with a well-powered meta-analysis. The majority of our GCIPL and RNFL-associated variants did not associate with POAG in a large consortium meta-analysis. Furthermore, Mendelian randomisation tests did not support a causal effect of GCIPL or RNFL thickness on POAG. This suggests that the major genetic processes that underlie variation in inner retinal thickness do not affect POAG. Mendelian randomisation in the reverse direction did provide some weak evidence for the genetic processes underlying POAG causing inner retinal thinning. Also expected was the very striking Mendelian randomisation evidence for higher IOP causing POAG. Put together, these findings suggest that the genetic causes of POAG are largely via raised IOP rather than processes underlying inner retinal thickness. As the thickness of these layers are consistently lower in individuals with POAG, this suggests that the change in thickness over time is a biomarker of the disease rather than the absolute level. This situation is similar to the observations that Hb1Ac is used as a biomarker for Type II diabetes but is confounded with red blood cell turnover and biology $[60,61]$. In both this example and the case of inner retinal thickness, aspects of the biomarker biology influenced by genetics may confound the thresholds of the biomarkers for clinical use. Indeed, the discovered loci, particularly the three loci associated with foveal hypoplasia, are likely to be confounders of the POAG biomarker. In the future it may be possible to adjust for the developmental baseline of these layers using genetic markers to provide more accurate metrics for POAG incidence and progression. The lack of an unambiguous glaucoma definition within the UK Biobank, and the fact that the age of glaucoma onset is relatively late in comparison to the mean age of the UK Biobank dataset, currently limits this work in this cohort. In addition, access to longitudinal data, to allow for monitoring of changes in thickness with disease progression would allow for analysis to interrogate this theory further. Currently only very small datasets of such nature are available, but in the future this type of data may help further elucidate the pathways of well-established POAG genes.

This study focused on two measurements from the inner retina, widely used in clinical practice. We have characterised the genetics underlying these traits, illuminating their role in known eye diseases, and explored some of the developmental processes around the fovea. The images from which the phenotypes are extracted are available and likely can be processed to a far richer representation. Improvements in image analysis using deep learning techniques [62] show great promise in this regard. We will extend this work both to the outer retina and to these richer phenotypes, increasing the breadth and detail of eye morphology that can be explained.

\section{Methods}

\section{Ethics statement}

The UK Biobank study was conducted with the approval of the North-West Research Ethics Committee (ref 06/MRE08/65), in accordance with the principles of the Declaration of 
Helsinki, and all participants gave written informed consent. This research has been conducted using the UK Biobank Resource under Application Number 2112.

In the case of the single cell expression study, all participants gave informed written consent [63], and the iPSC research was approved by the Human Research Ethics committees of the Royal Victorian Eye and Ear Hospital (11/1031H, 13/1151H-004), University of Melbourne (1545394), University of Tasmania (H0014124) and the University of Western Australia (RA/ $4 / 1 / 5255)$.

\section{UK Biobank cohort}

The UK Biobank is a large, well-studied prospective cohort sampled from across the UK at various sites. Participants completed a baseline questionnaire, physical measurements and provision of biological samples. The questionnaire collected information on demographics, anthropometrics, socioeconomic and lifestyle factors. This study was conducted between 2006 and 2010, recruiting more than half a million people aged 40-69 years old, identified via the NHS registry [64].

\section{Ophthalmic measurements and optical coherence tomography}

A subset of the cohort, comprising 132,041 participants, had further ocular data collected including IOP, visual acuity and autorefraction. As part of this ophthalmic assessment, 67,321 individuals underwent macular spectral domain OCT (SD-OCT) imaging.

OCT imaging. The SD-OCT imaging was done using the Topcon 3D OCT1000 Mark II and was performed following visual acuity, autorefraction and IOP measurements. The SD-OCT imaging was carried out in a dark room without pupil dilation using the 3-dimensional $6 \times 6 \mathrm{~mm}^{2}$ macular volume scan mode (512 A scans per B scan; 128 horizontal B scans in a raster pattern). The right eye was imaged first, and the scan was repeated for the left eye in most individuals.

Visual acuity measurement. Visual acuity was measured in both eyes using a logarithm of the minimum angle of resolution (LogMAR) chart (Precision Vision, LaSalle, Illinois, USA) displayed on a computer screen. The test was carried out with participants wearing their distance glasses at $4 \mathrm{~m}$, or at $1 \mathrm{~m}$ if a participant was unable to read letters at $4 \mathrm{~m}$. Participants were asked to read from the top of the chart and the test was terminated when two or more letters were read incorrectly. For patient-level visual acuity, we considered the value for the eye with better visual acuity.

Autorefraction measurement. Non-cycloplegic autorefraction was carried out using the Tomey RC-5000 Auto Refractor Keratometer (Tomey Corp., Nagoya, Japan). Up to 10 measurements were taken for each eye and the most reliable measure was automatically recorded. Spherical equivalent was calculated as spherical power plus half cylindrical power.

\section{Derivation of retinal thickness measures}

The OCT images were stored as both .fds files, a proprietary image storage file format, as well as as .dicom files. Version 1.6.1.1 of the Topcon Advanced Boundary Segmentation (TABS) algorithm [54] was applied to the images to segment the various retinal layers, and to calculate the thickness of such layers across the retinal fields, or across sub-fields as defined by the Early Treatment of Diabetic Retinopathy Study (ETDRS) [65] or Macula 6 grid [66] (Fig 1D). 


\section{Quality control and inclusion/exclusion criteria}

Participants were excluded from the study if they had withdrawn their consent, or were recommended for exclusion from genetics studies by UK Biobank (Fig 1C). Participants that were included represent the densest populated well-mixed population within the overall dataset. These participants were identified as being within a defined euclidean distance of the mean of the desired population, as selected by comparison to the HapMap Phase III study [67], in the principal component (PC) 1-PC2 space. Individuals were then excluded if they were related to third degree or more, based on the kinship information provided as a UK Biobank variable [68]. Further participants were removed from the dataset based on rigorous quality control of their OCT scans using previously implemented methods [30]. Briefly, this entailed all OCT images with an image quality score less than 45 being removed from the data set. A number of other segmentation indicators were also used as quality control metrics and for each of these, individuals representing the poorest $20 \%$ of the population in each of these measures were removed from the dataset. These segmentation indicators included: Inner Limiting Membrane (ILM) Indicator, a measure of the minimum localised edge strength around the ILM boundary across the entire scan. ILM indicator is useful for identifying blinks, scans that contain regions of severe signal fading, and segmentation errors; Valid count, a measure used to identify scans with a significant degree of clipping in the OCT scan's z-axis dimension; Minimum motion correlation, maximum motion delta and maximum motion factor, these indicators use both the nerve fibre layer and the full retinal thicknesses, from which Pearson correlations and absolute differences between the thickness data from each set of consecutive B-scans are calculated. The lowest correlation and the highest absolute difference in a scan are the resulting indicator scores and serve to identify blinks, eye motion artefacts, and segmentation failures. It

should be noted that the various indicators, including the image quality score, tend to be highly correlated with one another. The participants were further filtered to remove participants with outlier refractive error values defined as data points lying outside one standard deviation of 1.5 times the inter-quartile range from the median. We did not exclude participants with glaucoma from our analyses to avoid truncating the distribution of inner retinal thickness which would affect discovery power. The final dataset consisted of 31,434 participants.

\section{Genome-wide association study}

GWAS were performed using an additive linear model implemented using BGENIE v1.3 [68]. An individual GWAS was conducted for each of the two phenotypes, representing the thickness of the RNFL and GCIPL respectively, both averaged across the Macula 6 grid. Eye-specific covariates, namely refractive error, calculated from the spherical and cylindrical volume (refractive error $=$ spherical power $+0.5 \times$ cylindrical power), and technical covariates (Macula centre frame, Macula centre aline, ILM indicator, Valid count, Minimum motion correlation, Maximum Motion Delta, Maximum motion factor and Image quality) were regressed out of the thickness measurements for the separate eyes before the phenotypic measure of the mean across left and right eyes was calculated. Age, weight, height, sex, the ID of the OCT machine used in scan acquisition and the first 20 genotype PCs were then used as covariates in the model. SNPs were considered significantly associated if they met the genome-wide significance level $\left(\mathrm{P}<5 \times 10^{-8}\right)$. LD-score regression was implemented using LD SCore v1.0.1 [69].

\section{Discovered associated SNP set creation}

MTAG [33] was used to perform multi-trait meta-analysis across the GWAS summary statistics from the RNFL and GCIPL thickness analyses. This produced new adjusted P-values for each trait. For each SNP, the lowest P-value across the two traits was selected as the meta 
P-value. This was then used to plot a meta manhattan plot (Fig 2). GCTA Conditional and Joint Analysis (COJO) [70] was used to perform step-wise model selection to select significantly associated independent loci that were more than $10 \mathrm{Mb}$ apart. The resulting 46 loci constituted our discovered associated SNP set.

\section{Annotation of variants}

Each SNP in the discovered associated SNP set was manually annotated using both ENSEMBL [71] and the Open Targets Genetics [72] PHeWAS annotations. SNPs were further filtered and labelled as being within the same locus if they were within $1.5 \mathrm{Mb}$ of one another. This is represented in the shading of loci within Table 1, with loci considered to be within the same locus shaded the same colour, alternating grey and white.

\section{Replication of inner retinal morphology GWAS and examination of association with POAG}

Replication of the primary GWAS was sought in two datasets, the Raine Study, and the Rotterdam study.

The Raine Study is a multigenerational, longitudinal study based in Perth, Western Australia [73]. At the 20-year follow-up of the cohort, 1344 participants were enrolled in Raine Study Gen2, a cross-sectional study of eye diseases in young adults [74]. All participants underwent a standardised ocular examination, which included optical coherence tomography (OCT; Heidelberg Spectralis, Heidelberg Engineering GmbH, Heidelberg, Germany) measurements of the macula using a raster scan (31-line horizontal scan, $\left.30^{\circ} \times 25^{\circ}\right)$ centred on the fovea. Automatic segmentation was performed with the Spectralis software. Right and left eyes data were averaged for each subject. Genotyping was performed on 1593 participants, which included those who did not attend the vision assessments, via the Illumina Human $660 \mathrm{~W}$-quad BeadChip (Illumina, Inc., San Diego, CA, USA). After standard quality control, the cleaned genotypic datasets included 1495 individuals. Details of the quality control step have been previously described [75]. The mean macula RNFL (mRNFL) value was obtained by averaging the RNFL value on both eyes. We derived the GCIPL phenotype by summing up mean values of GCL and IPL. After removing individuals with missing phenotypes, the GWAS analyses on mRNFL ( $\mathrm{n}=1014)$ and GCIPL $(\mathrm{n}=1025)$ for the Raine cohort was conducted using Plink 2.00alpha. We adjusted the mean mRNFL values by the participant's average axial length (both eyes), fitted along with other standard covariates including age, sex and the top 5 ancestral principal components. The summary statistics for the relevant requested SNPs for replication were supplied back to the consortium.

The Rotterdam Study (RS) is a prospective population-based cohort study among individuals 45 years or older, residing in Ommoord, a district in Rotterdam, the Netherlands [76]. The first cohort started in January 1990 (RS, $1 \mathrm{n}=7983$ ). In February 2000 (RS2, $\mathrm{n}=3011$ ) a second and in February $2006(\mathrm{RS} 3, \mathrm{n}=3932)$ a third cohort was started. Follow-up examinations took place every 3 to 4 years. In September 2007, spectral-domain OCT scanning was added to the protocol. The current analysis comprises OCT data acquired at the fifth visit of the first cohort (RS1), the third visit of the second cohort (RS2), and the second visit of the third cohort (RS3). Eyes were initially scanned with the spectral-domain OCT-1000 Mark 2 (Topcon, Tokyo, Japan). From August 2011 onwards, this device was replaced with the spectral-domain OCT2000 owing to an update. As a result of this update about half of the RS2 cohort and all persons of RS3 cohort were examined on the OCT-2000 machine. The macula was scanned in the horizontal direction in an area of $6 \times 6 \times 1.68 \mathrm{~mm}$ with $512 \times 512 \times 480$ voxels (using OCT-1000) and $6 \times 6 \times 2.30 \mathrm{~mm}$ with $512 \times 512 \times 885$ voxels (using OCT-2000). Macula volumes scans 
were segmented using Iowa Reference Algorithms, version 3.6 (Retinal Image Analysis Lab, Iowa Institute for Biomedical Imaging, Iowa City, IA) (available at https://www.iibi.uiowa. edu/content/shared-software-download) [77]. Indices of quality control were used to preserve good-quality images and to exclude scans with segmentation errors. Scans included in our study had a segmentability index $>20 \%$ and an undefined region of $<20 \%$ (measures of segmentation/algorithm failures). If the scan of both eyes passed quality and control a random scan was chosen for the analysis. A total of 1000 persons in RS1, 1448 persons in RS2 and 765 persons in RS3 passed quality and control and were included in the study. The volume of the ganglion cell complex (RNFL + GCL + IPL) was calculated for 6x6mm macular surface and used as a quantitative phenotype in the GWAS. Genotyping was performed on either Illumina 550 (+duo) or Illumina 610 quad (Illumina, Inc., San Diego, CA, USA), genotypes were imputed to haplotype reference consortium (HRC) release 1 imputation panel, a detailed description of genotyping and pre- and post-imputation quality and control have been described elsewhere [78, 79]. For the GWAS, genotype and phenotype data were available for 899 persons in RS1, 1131 persons in RS2 and, 326 persons in RS3. We conducted a GWAS in PLINK v2.00 alpha and fitted a linear model adjusted for spherical equivalent, sex, age and the top 5 ancestral principal components [80]. As two different types of OCT machines were used during the data collection we initially analysed the cohorts separately and stratified by OCT machine. The results of these genome-wide analyses were then combined in a meta-analysis using METAL [81]. For every SNP we assessed the level of heterogeneity by calculating I2 values and Cochrans Q-test for heterogeneity as implemented in METAL.

\section{Sex-specific genetic effect on inner retinal morphology}

The genetic effect on the thickness of GCIPL and RNFL was linearly modelled for each of the SNPs in the discovered SNP set with an additional interaction term between the SNP and genetically determined sex included. Within the models, as with the GWAS, eye specific covariates were regressed out of the measurements for left and right eyes before a mean was taken across the eyes. The same covariates as used in the GWAS were also used in this linear model. An association was considered significant if $\mathrm{P}<0.05$ following Bonferroni correction.

\section{Higher dimensional detailed retinal phenotyping}

The surface of the inner limiting membrane (ILM) and bruchs membrane (BM) were segmented for each scan. To do so vectors of the columns, or axial scans, of an image slice were smoothed using a running median. Points of interest were identified as those outside two standard deviations of the median. The maximum and minimum of these points of interest were labelled as the ILM and BM, respectively. This method was iterated across each axial scan and outlier interpolation was applied to the composite layer boundary. For each image the matrix was calculated representing the distance between the ILM surface and the BM.

\section{Association with central foveal thickness and pigmentation}

For each genetic variant, the population was stratified by dosage of the alternative allele (where 0 represents homozygous reference, 1 represents heterozygous and 2 represents homozygous alternative). Means of the retinal thickness matrices, considering left and right eyes separately, were made across the participants within each genotype group resulting in the mean matrix per genotype per eye. Matrices of the difference in retinal thickness between homozygous reference and heterozygous, and homozygous reference and homozygous alternative, were calculated. Heatmaps of these difference matrices were plotted, with the colour representing the 
thickness of the retina. Additionally, a spline function is applied to the cross sectional vector along both axes of the models for each genotype and plotted as a line graph.

The overall thickness at the centre of the fovea was also calculated for the left eye of each individual. This was defined as the overall thickness of the retina at the midpoint of the two cross-sectional vectors, one across A-scans, one across B-scans, following application of a spline. These values were used as input to a linear model looking at the effect of each of the variants in the discovery set on the overall retinal thickness at the fovea. SNPs were considered to have a significant effect on the thickness of overall retinal thickness if $\mathrm{P}<0.05$ following Bonferroni correction for both retinal thickness values. The significant subset of SNPs were used in linear models of the SNP on hair and skin colour, as self-reported in the UK Biobank. In the case of hair colour, those with red hair were removed from the analysis. Both hair colour and skin colour were coded numerically, as in the UK Biobank, in ascending order from light to dark. SNPs were considered to have a significant effect on hair colour or skin colour if $\mathrm{P}<0.05$ after Bonferroni correction.

\section{Association with visual acuity}

The lead inner retina-association variants were tested for association with visual acuity. LogMAR visual acuity in the better seeing eye was used as the outcome measure, and the linear model run across all Europeans, as defined by genetic PCs $(n=100,818)$, and adjusted for age, sex, and the first 15 genetic PCs.

\section{Comparison of expression in different retinal cell types}

To investigate the expression profile of implicated genes in retinal organoids, we analysed data generated from a large cohort of induced pluripotent stem cell (iPSC)-derived cells. As previously described, a total of 247,520 cells from $110 \mathrm{iPSC}$ lines were profiled. In brief, retinal organoids were generated according to a previously established protocol [82]. Following this 49 day differentiation protocol, transcriptional profiling at single cell resolution was performed using the Chromium Single Cell 3 Library \& Gel bead kit (10x Genomics; PN-120237). Cells from the multi-donor single-cell suspensions were demultiplexed using transcriptome and genotype data with demuxlet v1.0 [83] and scrublet v0.20 [84]. Graph-based clustering via the Louvain algorithm that was implemented in Seurat was used to identify cell subpopulations $[85,86]$, with direct annotation of retinal cell types being performed using canonical eye-field and retinal marker genes.

\section{Mendelian randomisation analysis}

Mendelian randomisation analysis was undertaken using the TwoSampleMR package in $\mathrm{R}$ [87]. In the MR analysis, SNPs from the discovered associated SNP set were used as the exposure variable. Summary statistics for genetic association studies of IOP, were taken from [26] and when used as an exposure variable, were selected for genome-wide significance $(\mathrm{P}<5 \times$ $10^{-8}$ ). The internal LD pruning function was also applied to all exposure variables. POAG summary statistics were used from the POAG International Glaucoma Genetics Consortium (IGGC) meta-analysis [24]. The GCIPL and RNFL thickness (whether used as an exposure or an outcome) had the same set of technical covariates applied as the GWAS listed above. Eyespecific covariates, refractive error, calculated from the spherical and cylindrical volume (refractive error $=$ spherical power $+0.5 \times$ cylindrical power), and technical covariates (Macula centre frame, Macula centre aline, ILM indicator, Valid count, Minimum motion correlation, Maximum Motion Delta, Maximum motion factor and Image quality), were regressed out of the thickness measurements for the separate eyes before the phenotypic measure of the mean 
across left and right eyes was calculated. The association was applied with age, weight, height, sex, the ID of the OCT machine used in scan acquisition and the first 20 genotype PCs as covariates in the linear model. Although some of the associated SNP instruments were positive for these covariates (in particular height and weight) we considered these to be likely examples of horizontal pleiotropy. We used two meta-analysis methods, MR-Egger and inverse variance weighting (IVW); as the samples for the IOP consortium and the POAG consortium are independent of UK BioBank, the assumptions made by the MR-Egger scheme are valid.

\section{GCC thickness in the UK Biobank glaucoma population}

The cohort used in the GWAS was divided into those that had glaucoma $(\mathrm{n}=2,751)$, and those that did not $(n=28,314)$. Glaucoma case status was ascertained as participants who reported a history of glaucoma, glaucoma laser or glaucoma surgery on a touchscreen questionnaire or participants who had a glaucoma-related ICD 9/10 code on linked hospital episode statistic data (ICD 9: 365.*; ICD 10: H40.* [excluding H40.0], H42.*). GCC thickness, defined as the sum of GCIPL and RNFL thickness was calculated and a mean taken across the two eyes. A Wilcoxon rank sum test was performed to test for significant difference in GCC thickness between the two groups.

\section{Supporting information}

S1 Table. Comparison of biological characteristics between whole UK Biobank population with OCT data $(n=67,321)$, the group that passes our quality control criteria $(n=31,434)$, and the group that fails $(n=\mathbf{3 5 , 8 8 7})$. Results are presented as mean \pm standard deviation. (PDF)

S2 Table. Full GWAS summary statistics for $\mathbf{4 6}$ significant variants, including effect size, p-value and standard error (SE) for the individual phenotypes, GCIPL and RNFL (labelled accordingly), as well as the values selected within the meta-analysis (labelled "MTAG"). A1 is the effect allele.

(PDF)

S3 Table. Association of UK Biobank inner retinal thickness-associated variants with primary open-angle glaucoma in the International Glaucoma Genetics Consortium metaanalysis [1]. References [1] Gharahkhani, P. et al. Genome-wide meta-analysis identifies 127 open-angle glaucoma loci with consistent effect across ancestries. Nature Communications 12 (2021). URL https://pubmed.ncbi.nlm.nih.gov/33627673/.

S4 Table. Association of UK Biobank inner retinal thickness-associated variants with ganglion cell complex thickness in the Rotterdam study. Comparison between effect size and pvalues from meta-analysed inner retinal GWAS (labelled "MTAG"), and GWAS of the GCC thickness in the Rotterdam study (labelled "Rotterdam").

(PDF)

S5 Table. Association of UK Biobank inner retinal thickness-associated variants with retinal nerve fibre layer (RNFL) and ganglion cell inner plexiform layer (GCIPL) thickness in the Raine study. Comparison between effect size and p-values from meta-analysed inner retinal GWAS (labelled "MTAG"), and GWAS of the RNFL and GCIPL in the Raine Study (labelled "Raine RNFL" and "Raine GCIPL").

(PDF) 
S6 Table. Effect sizes and p-values for models of SNP effect on total retinal thickness at the fovea and visual acuity. LogMAR letters effect size refers to the difference in number of letters read on a standard LogMAR chart. A1 is the effect allele.

(PDF)

S7 Table. Results of gene set enrichment analysis using DEPICT [1]. The 30 most significant associations $(\mathrm{P}<0.05)$ are listed. References [1] Pers, T. H. et al. Biological interpretation of genome-wide association studies using predicted gene functions. Nature Communications $\mathbf{6}$, 1-9 (2015). URL www.nature.com/naturecommunications.

(PDF)

S8 Table. Results of tissue enrichment analysis using DEPICT [1]. All nominally significant associations $(\mathrm{P}<0.05)$ are listed. References [1] Pers, T. H. et al. Biological interpretation of genome-wide association studies using predicted gene functions. Nature Communications $\mathbf{6}$, 1-9 (2015). URL www.nature.com/naturecommunications.

(PDF)

S9 Table. Results of bidirectional two-sample Mendelian randomisation analysis between IOP and POAG, IOP and retinal layer thickness, and POAG and retinal layer thickness. Values reported for two meta-analysis methods, MR Egger and Inverse Variance Weighted. POAG summary statistics were taken from the POAG International Glaucoma Genetics Consortium (IGGC) meta-analysis [1]. Summary statistics for genetic association studies of IOP, were taken from [2]. References [1] Gharahkhani, P. et al. Genome-wide meta-analysis identifies 127 open-angle glaucoma loci with consistent effect across ancestries. Nature Communications 12 (2021). URL https://pubmed.ncbi.nlm.nih.gov/33627673/. [2] Khawaja, A. P. et al. Genome-wide analyses identify 68 new loci associated with intraocular pressure and improve risk prediction for primary open-angle glaucoma. Nature Genetics 50, 778-782 (2018). (PDF)

S1 Fig. Quantile-quantile plots of retinal thickness GWAS. (A) The quantile-quantile plot (qq-plot) for the GWAS of RNFL thickness prior to meta-analysis (Lambda GC = 1.11, Intercept $=1.01$, Ratio $=0.05$ ). (B) The qq-plot for the GWAS of GCIPL thickness prior to metaanalysis $($ Lambda $\mathrm{GC}=1.12$, Intercept $=1.01$, Ratio $=0.05)$.

(PDF)

S2 Fig. Sex-specific genetic effect of discovered loci. A boxplot of the mean thickness of the RNFL across the Macula 6 gird and across the left and right eyes stratified by genetically determined sex and genotype at rs146652416 (FOXG1). Genotype is encoded as homozygous reference (0), heterozygous (1) and homozygous alternative (2). A linear model of RNFL thickness including each discovered SNP and an interaction term between the SNP and genetically determined sex had a statistically significant sex-specific effect for rs 146652416 $\left(\mathrm{P}=8.08 \times 10^{-4}\right)$.

(PDF)

S3 Fig. Higher dimensional detailed retinal phenotyping. Mean cross-sectional models of overall retinal thickness across three groups defined by their allele state, homozygous reference, heterozygous or homozygous alternative at: (A) IGFBP3 (rs11762530), the variant with the lowest P-value aside from TSPAN10, as a control (B) TYR (rs1042602) (C) OCA2 (rs1800407) (D) TSPAN10 (rs7503894). The y axis is representative of total retinal thickness. (PDF) 
S4 Fig. Gene expression in retinal cell subpopulations. A heatmap of the mean expression of the genes labelled by the loci associated with inner retinal thickness in the different retinal cell subpopulations: singletons, retinal progenitor cells (RPC), retinal ganglion cells (RGC), interneurons, photoreceptors, retinal pigment epithelium (RPE) and lens. Expression values have been scaled and converted to z-scores.

(PDF)

S5 Fig. Mendelian randomisation of retinal layers and intraocular pressure. (A) Scatter plot of the relationship between the effect size of SNPs found significantly associated with GCIPL thickness, and the effect size of those SNPs on IOP. (B) Forest plot showing the effect size and direction of SNPs significantly associated with GCIPL thickness on IOP. (C) Scatter plot of the relationship between the effect size of SNPs found significantly associated with RNFL thickness, and the effect of those SNPs on IOP. (D) Forest plot showing the effect size and direction of SNPs significantly associated with RNFL thickness on IOP. (E) Scatter plot of the relationship between the effect size of SNPs found significantly associated in the meta-analysed GCIPL and RNFL thicknesses, and the effect of those SNPs on IOP. (F) Forest plot showing the effect size and direction of SNPs significantly associated in the meta-analysed GCIPL and RNFL thickness on IOP. Summary statistics for genetic association studies of IOP, were taken from [1]. References [1] Khawaja, A. P. et al. Genome-wide analyses identify 68 new loci associated with intraocular pressure and improve risk prediction for primary open-angle glaucoma. Nature Genetics 50, 778-782 (2018). (PDF)

S6 Fig. Mendelian randomisation of retinal layers and primary open angle glaucoma. (A) Scatter plot of the relationship between the effect size of SNPs found significantly associated with GCIPL thickness, and the effect size of those SNPs on POAG. (B) Forest plot showing the effect size and direction of SNPs significantly associated with GCIPL thickness on POAG. (C) Scatter plot of the relationship between the effect size of SNPs found significantly associated with RNFL thickness, and the effect of those SNPs on POAG. (D) Forest plot showing the effect size and direction of SNPs significantly associated with RNFL thickness on POAG. (E) Scatter plot of the relationship between the effect size of SNPs found significantly associated in the meta-analysed GCIPL and RNFL thicknesses, and the effect of those SNPs on POAG. (F) Forest plot showing the effect size and direction of SNPs significantly associated in the metaanalysed GCIPL and RNFL thickness on POAG. POAG summary statistics were taken from the POAG International Glaucoma Genetics Consortium (IGGC) meta-analysis [1]. Refrences [1] Gharahkhani, P. et al. Genome-wide meta-analysis identifies 127 open-angle glaucoma loci with consistent effect across ancestries. Nature Communications 12 (2021). URL https:// pubmed.ncbi.nlm.nih.gov/33627673/. (PDF)

S7 Fig. Mendelian randomisation of intraocular pressure and retinal layers. (A) Scatter plot of the relationship between the effect size of SNPs found significantly associated with IOP, and the effect size of those SNPs on GCIPL thickness. (B) Forest plot showing the effect size and direction of SNPs significantly associated with IOP on GCIPL thickness. (C) Scatter plot of the relationship between the effect size of SNPs found significantly associated with IOP, and the effect of those SNPs on RNFL thickness. (D) Forest plot showing the effect size and direction of SNPs significantly associated with IOP on RNFL thickness. (E) Scatter plot of the relationship between the effect size of SNPs found significantly associated with IOP and the effect size of those SNPs in the meta-analysed GCIPL and RNFL thickness. (F) Forest plot showing the effect size and direction of SNPs significantly associated with IOP on the meta-analysed 
GCIPL and RNFL thickness. Summary statistics for genetic association studies of IOP, were taken from [1]. References [1] Khawaja, A. P. et al. Genome-wide analyses identify 68 new loci associated with intraocular pressure and improve risk prediction for primary open-angle glaucoma. Nature Genetics 50, 778-782 (2018). (PDF)

S8 Fig. Mendelian randomisation of primary open angle glaucoma and retinal layers. (A) Scatter plot of the relationship between the effect size of SNPs found significantly associated with POAG, and the effect size of those SNPs on GCIPL thickness. (B) Forest plot showing the effect size and direction of SNPs significantly associated with POAG on GCIPL thickness. (C) Scatter plot of the relationship between the effect size of SNPs found significantly associated with POAG, and the effect of those SNPs on RNFL thickness. (D) Forest plot showing the effect size and direction of SNPs significantly associated with POAG on RNFL thickness. (E) Scatter plot of the relationship between the effect size of SNPs found significantly associated with POAG and the effect size of those SNPs in the meta-analysed GCIPL and RNFL thickness. (F) Forest plot showing the effect size and direction of SNPs significantly associated with POAG on the meta-analysed GCIPL and RNFL thickness. POAG summary statistics were taken from the POAG International Glaucoma Genetics Consortium (IGGC) meta-analysis [1]. Refrences [1] Gharahkhani, P. et al. Genome-wide meta-analysis identifies 127 openangle glaucoma loci with consistent effect across ancestries. Nature Communications 12 (2021). URL https://pubmed.ncbi.nlm.nih.gov/33627673/. (PDF)

S9 Fig. GCC thickness in the UK Biobank glaucoma population. A boxplot showing the thickness of the ganglion cell complex in the dataset comparing those who have glaucoma $(n=2,751)$, to those that do not $(n=28,314)$. An accompanying Wilcoxon rank sum test was performed that showed a statistically significant difference between the two populations $\left(\mathrm{P}=2.2 \times 10^{-16}\right)$. (PDF)

\section{Acknowledgments}

The authors are extremely grateful for the selfless participation of individuals in all cohorts used in this study (the UK Biobank, the Rotterdam Study, the Raine Study and the single cell expression study), and the staff managing these cohorts.

\section{Author Contributions}

Conceptualization: Hannah Currant, Ewan Birney, Anthony P. Khawaja.

Data curation: Anne Senabouth, Charles A. Reisman, Praveen J. Patel.

Formal analysis: Hannah Currant, Anne Senabouth.

Funding acquisition: Joseph E. Powell, Alice Pébay.

Project administration: Praveen J. Patel.

Resources: Maciej Daniszewski, Joseph E. Powell, Alice Pébay.

Supervision: Ewan Birney, Anthony P. Khawaja.

Validation: Puya Gharahkhani, Pieter W. M. Bonnemaijer, Alex W. Hewitt, Jason Charng, Jamie Craig, Caroline C. W. Klaver, Jue-Sheng Ong, Alberta A. H. J. Thiadens, Seyhan Yazar, Stuart MacGregor. 
Visualization: Hannah Currant, Anne Senabouth.

Writing - original draft: Hannah Currant, Ewan Birney, Anthony P. Khawaja.

Writing - review \& editing: Hannah Currant, Pirro Hysi, Tomas W. Fitzgerald, Puya Gharahkhani, Pieter W. M. Bonnemaijer, Alex W. Hewitt, Denize Atan, Tin Aung, Jason Charng, Hélène Choquet, Jamie Craig, Peng T. Khaw, Caroline C. W. Klaver, Michiaki Kubo, JueSheng Ong, Louis R. Pasquale, Charles A. Reisman, Mark J. Simcoe, Alberta A. H. J. Thiadens, Cornelia M. van Duijn, Seyhan Yazar, Eric Jorgenson, Stuart MacGregor, Chris J. Hammond, David A. Mackey, Janey L. Wiggs, Paul J. Foster, Praveen J. Patel, Ewan Birney, Anthony P. Khawaja.

\section{References}

1. Tick S.et al. Foveal Shape and Structure in a Normal Population. Investigative Opthalmology \& Visual Science 52, 5105 (2011). URL http://iovs.arvojournals.org/article.aspx?doi=10.1167/iovs.10-7005. https://doi.org/10.1007/s12015-017-9750-4 PMID: 28643176

2. Alamouti B. \& Funk J. Retinal thickness decreases with age: An OCT study. British Journal of Ophthalmology 87, 899-901 (2003). https://doi.org/10.1136/bjo.87.7.899

3. Khawaja A. P.et al. Comparison of Associations with Different Macular Inner Retinal Thickness Parameters in a Large Cohort: The UK Biobank. In Ophthalmology, vol. 127, 62-71 (Elsevier Inc., 2020).

4. Kelty P. J.et al. Macular Thickness Assessment in Healthy Eyes Based on Ethnicity Using Stratus OCT Optical Coherence Tomography. Investigative Opthalmology \& Visual Science 49, 2668 (2008). URL http://iovs.arvojournals.org/article.aspx?doi=10.1167/iovs.07-1000. https://doi.org/10.1007/s12015017-9750-4 PMID: 28643176

5. Pilat A. V., Proudlock F. A., Mohammad S. \& Gottlob I. Normal macular structure measured with optical coherence tomography across ethnicity. British Journal of Ophthalmology 98, 941-945 (2014). URL http://bjo.bmj.com/lookup/doi/10.1136/bjophthalmol-2013-303119 PMID: 24518076

6. Dai W.et al. Macular thickness profile and diabetic retinopathy: The Singapore Epidemiology of Eye Diseases Study. British Journal of Ophthalmology 102, 1072-1076 (2018). https://doi.org/10.1136/ bjophthalmol-2017-310959 PMID: 29175970

7. Goebel W. \& Kretzchmar-Gross T. Retinal thickness in diabetic retinopathy: A study using optical coherence tomography (OCT). Retina 22, 759-767 (2002). https://doi.org/10.1097/00006982-20021200000012 PMID: 12476103

8. Wood A.et al. Retinal and choroidal thickness in early age-related macular degeneration. American Journal of Ophthalmology 152 (2011). https://doi.org/10.1016/j.ajo.2011.05.021 PMID: 21851922

9. Nivison-Smith L., Wang H., Assaad N. \& Kalloniatis M. Retinal Thickness Changes throughout the Natural History of Drusen in Age-related Macular Degeneration. Optometry and Vision Science 95, 648-655 (2018). https://doi.org/10.1097/OPX.0000000000001256 PMID: 30063666

10. Herrero R.et al. Progressive degeneration of the retinal nerve fiber layer in patients with multiple sclerosis. Investigative Ophthalmology and Visual Science 53, 8344-8349 (2012). https://doi.org/10.1167/ iovs.12-10362 PMID: 23154461

11. Martinez-Lapiscina E. H.et al. Retinal thickness measured with optical coherence tomography and risk of disability worsening in multiple sclerosis: A cohort study. The Lancet Neurology 15, 574-584 (2016). https://doi.org/10.1016/S1474-4422(16)00068-5 PMID: 27011339

12. Ma L. J.et al. Progressive changes in the retinal structure of patients with Parkinson's disease. Journal of Parkinson's Disease 8, 85-92 (2018). https://doi.org/10.3233/JPD-171184 PMID: 29480221

13. den Haan J., Verbraak F. D., Visser P. J. \& Bouwman F. H. Retinal thickness in Alzheimer's disease: A systematic review andÂ meta-analysis. Alzheimer's and Dementia: Diagnosis, Assessment and Disease Monitoring 6, 162-170 (2017). https://doi.org/10.1016/j.dadm.2016.12.014 PMID: 28275698

14. Kim J.-I. \& Kang B.-H. Decreased retinal thickness in patients with Alzheimer's disease is correlated with disease severity. PLOS ONE 14, e0224180 (2019). URL https://dx.plos.org/10.1371/journal.pone. 0224180 PMID: 31689310

15. Lee W. W., Tajunisah I., Sharmilla K., Peyman M. \& Subrayan V. Retinal nerve fiber layer structure abnormalities in schizophrenia and its relationship to disease state: Evidence from optical coherence tomography. Investigative Ophthalmology and Visual Science 54, 7785-7792 (2013). https://doi.org/ 10.1167/iovs.13-12534 PMID: 24135757 
16. Yilmaz U.et al. Retinal nerve fiber layer and macular thickness measurement in patients with schizophrenia. European Journal of Ophthalmology 26, 375-378 (2016). https://doi.org/10.5301/ejo.5000723 PMID: 26742871

17. King A., Azuara-Blanco A. \& Tuulonen A. Glaucoma. BMJ (Clinical research ed.) 346, f3518 (2013). URL http://www.ncbi.nlm.nih.gov/pubmed/23757737.

18. Tatham A. J. \& Medeiros F. A. Detecting Structural Progression in Glaucoma with Optical Coherence Tomography. Ophthalmology 124, S57-S65 (2017). URL https://www.sciencedirect.com/science/ article/pii/S0161642017305146?via\%3Dihub. https://doi.org/10.1016/j.ophtha.2017.07.015 PMID: 29157363

19. Shin J. W., Sung K. R., Lee G. C., Durbin M. K. \& Cheng D. Ganglion Cell-Inner Plexiform Layer Change Detected by Optical Coherence Tomography Indicates Progression in Advanced Glaucoma. Ophthalmology 124, 1466-1474 (2017). https://doi.org/10.1016/j.ophtha.2017.04.023 PMID: 28549518

20. Hood D. C. Improving our understanding, and detection, of glaucomatous damage: An approach based upon optical coherence tomography (OCT). Progress in Retinal and Eye Research 57, 46-75 (2017). URL http://dx.doi.org/10.1016/j.preteyeres.2016.12.002 PMID: 28012881

21. Gibson J.et al. Genome-wide association study of primary open angle glaucoma risk and quantitative traits. Molecular Vision 18, 1083-1092 (2012). PMID: 22605921

22. Choquet H.et al. A multiethnic genome-wide association study of primary open-angle glaucoma identifies novel risk loci. Nature Communications 9, 1-14 (2018). https://doi.org/10.1038/s41467-01804555-4 PMID: 29891935

23. Choquet H., Wiggs J. L. \& Khawaja A. P. Clinical implications of recent advances in primary open-angle glaucoma genetics (2020). URL https://pubmed.ncbi.nlm.nih.gov/31645673/.

24. Gharahkhani P.et al. Genome-wide meta-analysis identifies 127 open-angle glaucoma loci with consistent effect across ancestries. Nature Communications 12 (2021). URL https://pubmed.ncbi.nlm.nih. gov/33627673/. https://doi.org/10.1038/s41467-020-20851-4

25. Choquet $\mathrm{H}$.et al. A large multi-ethnic genome-wide association study identifies novel genetic loci for intraocular pressure. Nature Communications 8 (2017). URL https://pubmed.ncbi.nlm.nih.gov/ 29235454/. https://doi.org/10.1038/s41467-017-01913-6

26. Khawaja A. P.et al. Genome-wide analyses identify 68 new loci associated with intraocular pressure and improve risk prediction for primary open-angle glaucoma. Nature Genetics 50, 778-782 (2018). https://doi.org/10.1038/s41588-018-0126-8 PMID: 29785010

27. Y. Liu, Ph D., Allingham R. R. \& M D. Major review: Molecular genetics of primary open-angle glaucoma. Experimental Eye Research 160, 62-84 (2017). URL http://dx.doi.org/10.1016/j.exer.2017.05. 002

28. Ryoo N.-K. et al. Thickness of retina and choroid in the elderly population and its association with Complement Factor H polymorphism: KLoSHA Eye study. PLOS ONE 13, e0209276 (2018). URL http://dx. plos.org/10.1371/journal.pone.0209276 PMID: 30596689

29. Gao X. R., Huang H. \& Kim H. Genome-wide association analyses identify 139 loci associated with macular thickness in the UK Biobank cohort. Human Molecular Genetics 28, 1162-1172 (2019). https://doi.org/10.1093/hmg/ddy422 PMID: 30535121

30. Patel P. J.et al. Spectral-domain optical coherence tomography imaging in 67321 adults: Associations with macular thickness in the UK biobank study. Ophthalmology 123, 829-840 (2016). URL http://dx. doi.org/10.1016/j.ophtha.2015.11.009 PMID: 26746598

31. Sudlow C.et al. UK Biobank: An Open Access Resource for Identifying the Causes of a Wide Range of Complex Diseases of Middle and Old Age. PLOS Medicine 12, e1001779 (2015). URL https://dx.plos. org/10.1371/journal.pmed.1001779 PMID: 25826379

32. Mota Á. et al. Isolated Foveal Hypoplasia: Tomographic, Angiographic and Autofluorescence Patterns. Case Reports in Ophthalmological Medicine 2012, 1-3 (2012). URL/pmc/articles/PMC3415089/? report=abstracthttps://www.ncbi.nlm.nih.gov/pmc/articles/PMC3415089/. https://doi.org/10.1155/2012/ 864958 PMID: 22900218

33. Turley P.et al. Multi-trait analysis of genome-wide association summary statistics using MTAG. Nature Genetics 50, 229-237 (2018). https://doi.org/10.1038/s41588-017-0009-4 PMID: 29292387

34. Tomita Y. \& Suzuki T. Genetics of pigmentary disorders (2004).

35. Ariani F.et al. FOXG1 Is Responsible for the Congenital Variant of Rett Syndrome. American Journal of Human Genetics 83, 89-93 (2008). URL/pmc/articles/PMC2443837//pmc/articles/PMC2443837/? report=abstracthttps://www.ncbi.nlm.nih.gov/pmc/articles/PMC2443837/. https://doi.org/10.1016/j. ajhg.2008.05.015 PMID: 18571142 
36. Macgregor S. et al. Genome-wide association identifies ATOH7 as a major gene determining human optic disc size URL http://blocks.fhcrc.org/.

37. Choy S. W.et al. A cascade of irx1a and irx2a controls shh expression during retinogenesis. Developmental Dynamics 239, 3204-3214 (2010). URL https://pubmed.ncbi.nlm.nih.gov/21046643/. https:// doi.org/10.1002/dvdy.22462

38. Maksimova N.et al. Neuroblastoma amplified sequence gene is associated with a novel short stature syndrome characterised by optic nerve atrophy and Pelger-Huët anomaly. Journal of Medical Genetics 47, 538-548 (2010). URL https://pubmed.ncbi.nlm.nih.gov/20577004/. https://doi.org/10.1136/jmg. 2009.074815

39. O'Sullivan M. L.et al. Astrocytes follow ganglion cell axons to establish an angiogenic template during retinal development. GLIA 65, 1697-1716 (2017). URL https://pubmed.ncbi.nlm.nih.gov/28722174/. https://doi.org/10.1002/glia.23189

40. Jen J. C.et al. Mutations in a human ROBO gene disrupt hindbrain axon pathway crossing and morphogenesis. Science 304, 1509-1513 (2004). URL https://pubmed.ncbi.nlm.nih.gov/15105459/. https:// doi.org/10.1126/science.1096437

41. Mattar P. \& Cayouette M. Mechanisms of temporal identity regulation in mouse retinal progenitor cells URL http://dx.doi.org/10.1080/23262133.2015.1125409.

42. Takata N.et al. An Eye Organoid Approach Identifies Six3 Suppression of R-spondin 2 as a Critical Step in Mouse Neuroretina Differentiation. Cell Reports 21, 1534-1549 (2017). URL https://pubmed.ncbi. nlm.nih.gov/29117559/. https://doi.org/10.1016/j.celrep.2017.10.041

43. Hysi P. G.et al. Meta-analysis of 542,934 subjects of European ancestry identifies new genes and mechanisms predisposing to refractive error and myopia. Nature Genetics 52, 401-407 (2020). URL https://pubmed.ncbi.nlm.nih.gov/32231278/. https://doi.org/10.1038/s41588-020-0599-0

44. Verhoeven V. J.et al. Genome-wide meta-analyses of multiancestry cohorts identify multiple new susceptibility loci for refractive error and myopia. Nature Genetics 45, 314-318 (2013). URL https:// pubmed.ncbi.nlm.nih.gov/23396134/https://pubmed.ncbi.nlm.nih.gov/23396134/?dopt=Abstract. https://doi.org/10.1038/ng.2554

45. Meyer C. H., Lapolice D. J. \& Freedman S. F. Foveal hypoplasia in oculocutaneous albinism demonstrated by optical coherence tomography. American journal of ophthalmology 133, 409-10 (2002). URL http://www.ncbi.nlm.nih.gov/pubmed/11860983. https://doi.org/10.1016/S0002-9394(01)01326-5

46. Iotchkova V. et al. GARFIELD—GWAS Analysis of Regulatory or Functional Information Enrichment with LD correction (2016). URL https://doi.org/10.1101/085738.

47. Pers T. H.et al. Biological interpretation of genome-wide association studies using predicted gene functions. Nature Communications 6, 1-9 (2015). URL www.nature.com/naturecommunications. https:// doi.org/10.1038/ncomms6890 PMID: 25597830

48. Garway-Heath D. F.et al. Latanoprost for open-angle glaucoma (UKGTS): A randomised, multicentre, placebo-controlled trial. The Lancet 385, 1295-1304 (2015). https://doi.org/10.1016/S0140-6736(14) 62111-5 PMID: 25533656

49. Kim K. E. \& Park K. H. Macular imaging by optical coherence tomography in the diagnosis and management of glaucoma (2018). URL http://www.ncbi.nlm.nih.gov/pubmed/29055905.

50. Kansal V., Armstrong J. J., Pintwala R. \& Hutnik C. Optical coherence tomography for glaucoma diagnosis: An evidence based meta-analysis. PLOS ONE 13, e0190621 (2018). URL http://www.ncbi.nlm. nih.gov/pubmed/29300765http://www.pubmedcentral.nih.gov/articlerender.fcgi?artid=PMC5754143. https://doi.org/10.1371/journal.pone.0190621

51. Ulmer Carnes M.et al. Discovery and Functional Annotation of SIX6 Variants in Primary Open-Angle Glaucoma. PLoS Genetics 10 (2014). URL https://pubmed.ncbi.nlm.nih.gov/24875647/. https://doi.org/ 10.1371/journal.pgen.1004372

52. Grønskov K., Ek J. \& Brondum-Nielsen K. Oculocutaneous albinism. Orphanet Journal of Rare Diseases 2, 43 (2007). URL http://ojrd.biomedcentral.com/articles/10.1186/1750-1172-2-43 PMID: 17980020

53. Liu F.et al. Digital Quantification of Human Eye Color Highlights Genetic Association of Three New Loci. PLoS Genetics 6, e1000934 (2010). URL https://dx.plos.org/10.1371/journal.pgen.1000934 PMID: 20463881

54. Yang Q.et al. Automated layer segmentation of macular OCT images using dual-scale gradient information. Optics Express 18, 21293 (2010). https://doi.org/10.1364/OE.18.021293 PMID: 20941025

55. Tao R.et al. Perifovea retinal thickness as an ophthalmic biomarker for mild cognitive impairment and early Alzheimer's disease. Alzheimer's and Dementia: Diagnosis, Assessment and Disease Monitoring 11, 405-414 (2019). URL https://pubmed.ncbi.nlm.nih.gov/31206006/. https://doi.org/10.1016/j.dadm. 2019.04.003 
56. Costello F. \& Burton J. M. Retinal imaging with optical coherence tomography: A biomarker in multiple sclerosis? (2018). URL https://pubmed.ncbi.nlm.nih.gov/30104912/.

57. Deva R.et al. Vision-threatening retinal abnormalities in chronic kidney disease stages 3 to 5 . Clinical Journal of the American Society of Nephrology 6, 1866-1871 (2011). https://doi.org/10.2215/CJN. 10321110 PMID: 21784818

58. Bodaghi B., Massamba N. \& Izzedine H. The eye: A window on kidney diseases. Clinical Kidney Journal 7, 337-338 (2014). https://doi.org/10.1093/ckj/sfu073 PMID: 25852906

59. Savige J., Ratnaike S. \& Colville D. Retinal abnormalities characteristic of inherited renal disease. Journal of the American Society of Nephrology 22, 1403-1415 (2011). https://doi.org/10.1681/ASN. 2010090965 PMID: 21372206

60. Wheeler E.et al. Impact of common genetic determinants of Hemoglobin A1c on type 2 diabetes risk and diagnosis in ancestrally diverse populations: A transethnic genome-wide meta-analysis. PLoS medicine 14, e1002383 (2017). URL https://dx.plos.org/10.1371/journal.pmed.1002383http://www. ncbi.nlm.nih.gov/pubmed/28898252http://www.pubmedcentral.nih.gov/articlerender.fcgi?artid= PMC5595282. https://doi.org/10.1371/journal.pmed.1002383

61. Leong A.et al. Mendelian Randomization Analysis of Hemoglobin A1c as a Risk Factor for Coronary Artery Disease. Diabetes care 42, 1202-1208 (2019). URL http://care.diabetesjournals.org/lookup/doi/ 10.2337/dc18-1712http://www.ncbi.nlm.nih.gov/pubmed/30659074http://www.pubmedcentral.nih.gov/ articlerender.fcgi?artid=PMC6609962. https://doi.org/10.2337/dc18-1712

62. De Fauw J.et al. Clinically applicable deep learning for diagnosis and referral in retinal disease. Nature Medicine 24, 1342-1350 (2018). URL http://www.nature.com/articles/s41591-018-0107-6 PMID: 30104768

63. McCaughey T. et al. An Interactive Multimedia Approach to Improving Informed Consent for Induced Pluripotent Stem Cell Research (2016). URL https://pubmed.ncbi.nlm.nih.gov/26942850/.

64. UK Biobank. UK Biobank: Protocol for a large-scale prospective epidemiological resource. Tech. Rep. March (2007). URL https://www.ukbiobank.ac.uk/wp-content/uploads/2011/11/UK-Biobank-Protocol. pdf.

65. Camparini M., Cassinari P., Ferrigno L. \& Macaluso C. Investigative ophthalmology \& visual science., vol. 42 ( C.V. Mosby Co, 2001). URL https://iovs. arvojournals.org/article.aspx?articleid=2162384.

66. Chaglasian M.et al. The development of a reference database with the Topcon 3D OCT-1 Maestro. Clinical Ophthalmology 12, 849-857 (2018). URL/pmc/articles/PMC5944450/?report=abstracthttps:// www.ncbi.nlm.nih.gov/pmc/articles/PMC5944450/. https://doi.org/10.2147/OPTH.S155229 PMID: 29765199

67. Altshuler D. M.et al. Integrating common and rare genetic variation in diverse human populations. Nature 467, 52-58 (2010). URL https://www.nature.com/articles/nature09298. https://doi.org/10.1038/ nature09298 PMID: 20811451

68. Bycroft C.et al. The UK Biobank resource with deep phenotyping and genomic data. Nature 562, 203209 (2018). https://doi.org/10.1038/s41586-018-0579-z PMID: 30305743

69. Bulik-Sullivan B.et al. LD score regression distinguishes confounding from polygenicity in genome-wide association studies. Nature Genetics 47, 291-295 (2015). URL https://www.nature.com/articles/ng. 3211. https://doi.org/10.1038/ng.3211 PMID: 25642630

70. Yang J.et al. Conditional and joint multiple-SNP analysis of GWAS summary statistics identifies additional variants influencing complex traits. Nature Genetics 44, 369-375 (2012). https://doi.org/10.1038/ ng.2213 PMID: 22426310

71. Cunningham F.et al. Ensembl 2019. Nucleic Acids Research 47, D745-D751 (2019). URL https:// academic.oup.com/nar/article/47/D1/D745/5165265. https://doi.org/10.1093/nar/gky1113 PMID: 30407521

72. Carvalho-Silva D.et al. Open Targets Platform: new developments and updates two years on. Nucleic Acids Research 47, D1056-D1065 (2019). URL https://academic.oup.com/nar/article/47/D1/D1056/ 5193331. https://doi.org/10.1093/nar/gky1133 PMID: 30462303

73. McKnight C. M.et al. Birth of a cohort - The first 20 years of the raine study. Medical Journal of Australia 197, 608-610 (2012). URL https://pubmed.ncbi.nlm.nih.gov/23230915/. https://doi.org/10.5694/ mja12.10698

74. Yazar S.et al. Raine eye health study: Design, methodology and baseline prevalence of ophthalmic disease in a birth-cohort study of young adults. Ophthalmic Genetics 34, 199-208 (2013). URL https:// pubmed.ncbi.nlm.nih.gov/23301674/. https://doi.org/10.3109/13816810.2012.755632

75. Yazar S.et al. Genetic and environmental factors in conjunctival UV autofluorescence. JAMA Ophthalmology 133, 406-412 (2015). URL/pmc/articles/PMC4840232/?report=abstracthttps://www.ncbi.nlm. 
nih.gov/pmc/articles/PMC4840232/. https://doi.org/10.1001/jamaophthalmol.2014.5627 PMID: 25590795

76. Hofman A.et al. The Rotterdam Study: 2016 objectives and design update. European Journal of Epidemiology 30, 661-708 (2015). URL https://pubmed.ncbi.nlm.nih.gov/26386597/. https://doi.org/10. 1007/s10654-015-0082-x

77. Sonka M. \& Abràmoff M. D. Quantitative analysis of retinal OCT (2016).

78. McCarthy S.et al. A reference panel of 64,976 haplotypes for genotype imputation. Nature Genetics 48 , 1279-1283 (2016). URL https://www.nature.com/articles/ng.3643. https://doi.org/10.1038/ng.3643 PMID: 27548312

79. Bonnemaijer P. W.et al. Multi-trait genome-wide association study identifies new loci associated with optic disc parameters. Communications Biology 2, 1-12 (2019). URL https://www.nature.com/articles/ s42003-019-0634-9. https://doi.org/10.1038/s42003-019-0634-9 PMID: 31798171

80. Chang C. C.et al. Second-generation PLINK: rising to the challenge of larger and richer datasets. GigaScience 4, 7 (2015). URL https://academic.oup.com/gigascience/article-lookup/doi/10.1186/s13742015-0047-8 PMID: 25722852

81. Willer C. J., Li Y. \& Abecasis G. R. METAL: fast and efficient meta-analysis of genomewide association scans. BIOINFORMATICS APPLICATIONS NOTE 26, 2190-2191 (2010). URL http://www.sph.umich. edu/csg/abecasis/metal/. https://doi.org/10.1093/bioinformatics/btq340 PMID: 20616382

82. Reichman S.et al. Generation of Storable Retinal Organoids and Retinal Pigmented Epithelium from Adherent Human iPS Cells in Xeno-Free and Feeder-Free Conditions. Stem Cells 35, 1176-1188 (2017). URL https://pubmed.ncbi.nlm.nih.gov/28220575/. https://doi.org/10.1002/stem.2586

83. Kang H. M.et al. Multiplexed droplet single-cell RNA-sequencing using natural genetic variation. Nature Biotechnology 36, 89-94 (2018). URL https://pubmed.ncbi.nlm.nih.gov/29227470/. https://doi.org/10. 1038/nbt.4042

84. Wolock S. L., Lopez R. \& Klein A. M. Scrublet: Computational Identification of Cell Doublets in SingleCell Transcriptomic Data. Cell Systems 8, 281-291.e9 (2019). URL https://pubmed.ncbi.nlm.nih.gov/ 30954476/. https://doi.org/10.1016/j.cels.2018.11.005

85. Levine J. H.et al. Data-Driven Phenotypic Dissection of AML Reveals Progenitor-like Cells that Correlate with Prognosis. Cell 162, 184-197 (2015). URL https://pubmed.ncbi.nlm.nih.gov/26095251/. https://doi.org/10.1016/j.cell.2015.05.047

86. Blondel V. D., Guillaume J. L., Lambiotte R. \& Lefebvre E. Fast unfolding of communities in large networks. Journal of Statistical Mechanics: Theory and Experiment 2008, P10008 (2008). URL https:// iopscience.iop.org/article/10.1088/1742-5468/2008/10/P10008https://iopscience.iop.org/article/10. 1088/1742-5468/2008/10/P10008/meta.0803.0476

87. Hemani G.et al. The MR-base platform supports systematic causal inference across the human phenome. eLife 7 (2018). https://doi.org/10.7554/eLife.34408 PMID: 29846171 


\section{University Library}

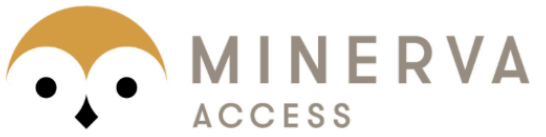

A gateway to Melbourne's research publications

Minerva Access is the Institutional Repository of The University of Melbourne

\section{Author/s:}

Currant, H;Hysi, P;Fitzgerald, TW;Gharahkhani, P;Bonnemaijer, PWM;Senabouth, A;Hewitt, AW;Atan, D;Aung, T;Charng, J;Choquet, H;Craig, J;Khaw, PT;Klaver, CCW;Kubo, M;Ong, JS;Pasquale, LR;Reisman, CA;Daniszewski, M;Powell, JE;Pebay, A;Simcoe, MJ;Thiadens, AAHJ;van Duijn, CM;Yazar, S;Jorgenson, E;MacGregor, S;Hammond, CJ;Mackey, DA;Wiggs, JL;Foster, PJ;Patel, PJ;Birney, E;Khawaja, AP

Title:

Genetic variation affects morphological retinal phenotypes extracted from UK Biobank optical coherence tomography images

Date:

2021-05-01

\section{Citation:}

Currant, H., Hysi, P., Fitzgerald, T. W., Gharahkhani, P., Bonnemaijer, P. W. M., Senabouth, A., Hewitt, A. W., Atan, D., Aung, T., Charng, J., Choquet, H., Craig, J., Khaw, P. T., Klaver, C. C. W., Kubo, M., Ong, J. -S., Pasquale, L. R., Reisman, C. A., Daniszewski, M. ,... Khawaja, A. P. (2021). Genetic variation affects morphological retinal phenotypes extracted from UK Biobank optical coherence tomography images. PLOS GENETICS, 17 (5), https:// doi.org/10.1371/journal.pgen. 1009497.

Persistent Link:

http://hdl.handle.net/11343/278329

License:

CC BY 